Open Access

\title{
Post-mortem brain analyses of the Lothian Birth Cohort 1936: extending lifetime cognitive and brain phenotyping to the level of the synapse
}

Christopher M. Henstridge ${ }^{1}$, Rosemary J. Jackson ${ }^{1}$, JeeSoo M. Kim ${ }^{1}$, Abigail G. Herrmann ${ }^{1}$, Ann K. Wright ${ }^{2}$, Sarah E. Harris 3,4, Mark E. Bastin, 3,5, John M. Starr 3,6, Joanna Wardlaw'3,5, Thomas H. Gillingwater ${ }^{2}$, Colin Smith ${ }^{3,5,8}$, Chris-Anne McKenzie ${ }^{5}$, Simon R. Cox ${ }^{3,7}$, lan J. Deary ${ }^{3,7^{*}+}$ and Tara L. Spires-Jones ${ }^{1,8^{*}+}$

\begin{abstract}
Introduction: Non-pathological, age-related cognitive decline varies markedly between individuals and places significant financial and emotional strain on people, their families and society as a whole. Understanding the differential age-related decline in brain function is critical not only for the development of therapeutics to prolong cognitive health into old age, but also to gain insight into pathological ageing such as Alzheimer's disease. The Lothian Birth Cohort of 1936 (LBC1936) comprises a rare group of people for whom there are childhood cognitive test scores and longitudinal cognitive data during older age, detailed structural brain MRI, genome-wide genotyping, and a multitude of other biological, psycho-social, and epidemiological data. Synaptic integrity is a strong indicator of cognitive health in the human brain; however, until recently, it was prohibitively difficult to perform detailed analyses of synaptic and axonal structure in human tissue sections. We have adapted a novel method of tissue preparation at autopsy to allow the study of human synapses from the LBC1936 cohort in unprecedented morphological and molecular detail, using the high-resolution imaging techniques of array tomography and electron microscopy. This allows us to analyze the brain at sub-micron resolution to assess density, protein composition and health of synapses. Here we present data from the first donated LBC1936 brain and compare our findings to Alzheimer's diseased tissue to highlight the differences between healthy and pathological brain ageing.
\end{abstract}

Results: Our data indicates that compared to an Alzheimer's disease patient, the cognitively normal LBC1936 participant had a remarkable degree of preservation of synaptic structures. However, morphological and molecular markers of degeneration in areas of the brain associated with cognition (prefrontal cortex, anterior cingulate cortex, and superior temporal gyrus) were observed.

Conclusions: Our novel post-mortem protocol facilitates high-resolution neuropathological analysis of the wellcharacterized LBC1936 cohort, extending phenotyping beyond cognition and in vivo imaging to now include neuro pathological changes, at the level of single synapses. This approach offers an unprecedented opportunity to study synaptic and axonal integrity during ageing and how it contributes to differences in age-related cognitive change.

\footnotetext{
* Correspondence: I.Deary@ed.ac.uk; Tara.spires-jones@ed.ac.uk

${ }^{\dagger}$ Equal contributors

${ }^{3}$ Centre for Cognitive Ageing and Cognitive Epidemiology, University of

Edinburgh, 7 George Square, Edinburgh EH8 9JZ, UK

${ }^{1}$ Centre for Cognitive and Neural Systems, University of Edinburgh, 1 George

Square, Edinburgh EH8 9JZ, UK

Full list of author information is available at the end of the article
}

\section{Biomed Central}

(c) 2015 Henstridge et al. Open Access This article is distributed under the terms of the Creative Commons Attribution 4.0 International License (http://creativecommons.org/licenses/by/4.0/), which permits unrestricted use, distribution, and reproduction in any medium, provided you give appropriate credit to the original author(s) and the source, provide a link to the Creative Commons license, and indicate if changes were made. The Creative Commons Public Domain Dedication waiver (http://creativecommons.org/publicdomain/zero/1.0/) applies to the data made available in this article, unless otherwise stated. 


\section{Introduction}

The global number of older, dependent people is projected to reach 277 million by 2050 [1]. According to government figures, more than one-in-four UK inhabitants will be aged 65 or over by the same year [2]. Cognitive decline is a feature of ageing, yet its severity varies dramatically between individuals and a deeper understanding of the underlying factors influencing brain ageing, may render this process open to modification. An important question to address is whether the brain changes observed in healthy older individuals are distinct from changes observed in neuropathologies such as Alzheimer's disease (AD). For example, synapse loss, amyloid plaques and neurofibrillary tangles are the hallmarks of $\mathrm{AD}$, yet these features can also be observed in the brains of cognitivelyhealthy older people [3]. However, before we can assess the influence of pathways or proteins in pathological cognitive decline we need a greater understanding of the normal ageing process [4].

Normal cognitive ageing refers to age-related cognitive change with an absence of disease and is thought to be due to a number of underlying factors [4]. As people age, most tissues and bodily functions begin to decline and neurological function is not exempt from this effect. In fact, some age-dependent gene expression changes are evolutionary conserved throughout the animal kingdom from humans to nematode worms, including genes involved in the stress response, mitochondrial function and the immune response [3]. In the brain, changes in neuron number and neurite complexity occur, glial cells become more active, neurotransmitter levels are altered, pigments and proteins begin to accumulate in cells and neurovascular changes become increasingly prevalent [5]. With the introduction of functional MR imaging, a systems view of neuronal activity could be observed for the first time, during the course of brain ageing. This revealed a loss of regional co-ordination in aged brains during higher order cognitive tasks [6]. These effects may be due in part, to altered axonal and/or myelin physiology, as increased incidence of white matter hyperintensities appear to correlate with more severe cognitive decline [7]. However, synapses are now increasingly thought to be essential in the state of cognitive ageing [8]. During normal ageing the brain transcriptome alters and many agerelated changes occur in genes involved in synaptic function $[9,10]$. Indeed, data from ageing cohorts have revealed that higher levels of presynaptic markers correlate with better cognitive function [11]. Furthermore, a recent UK-based cohort study revealed that genetic variability within synaptic genes, contributes to the variability in general intelligence [12]. These findings add to a growing body of literature suggesting synapses are not only critical for healthy cognitive-ageing, but represent the first neuronal feature to be affected in $\mathrm{AD}[13,14]$.
Large cohort studies that trace cognition through to brain changes at death are a powerful tool for determining the neurobiological contributors to cognitive ageing. Several such studies including the Religious Orders Study and the Rush Memory and Ageing Project have assessed cognitive change in old age and characterized neural tissue postmortem, providing a wealth of information on potential factors and correlates that may influence elderly cognitive change [15]. However, most ageing studies lack the longitudinal data on individual participant's cognitive performance from childhood to adulthood which is important for distinguishing which changes correlate with cognitive variation in age are truly age-related as opposed to being effects of other factors such as genetics [16].

The Lothian Birth Cohort of 1936 (LBC1936) is a sample of 1091 people for whom we have childhood cognitive test scores, longitudinal cognitive data during ageing, detailed structural MRI, genome-wide genotyping, and a multitude of other biological and epidemiological data [17]. Furthermore, 173 participants of the cohort have agreed to donate their brains to the study for detailed post-mortem analysis. The cohort's data have been used to examine determinants of cognitive change between childhood and age 70, and within older age. The LBC1936 research team refers to the age 11 to age 70 cognitive ageing as lifetime cognitive ageing, and their candidate determinants include genetic, lifestyle, psychosocial and biomedical (including brain imaging factors). The following factors make small contributions to cognitive efficiency in older age after adjusting for childhood cognitive function (which effectively means they contribute positively to lifetime cognitive ageing): being bilingual [18], attaining higher education, [19], having had a more complex occupation [20], having more social support and being less lonely [21], being physically more active [22], having a lower genetic risk of schizophrenia [23] and lacking the e4 allele of the gene for APOE [24]. The LBC1936 study has found several instances in which cross-sectional associations between putative determinants of cognitive ageing and cognitive test scores at age 70 are almost wholly confounded by childhood intelligence test scores. Thus, engaging more in socio-intellectual activities [22], drinking more red wine at moderate levels [25], having a lower body mass index [26], eating a Mediterranean diet [27], having lower blood levels of C-reactive protein [28] and not being infected by the cytomegalovirus [29] are all associated with better cognitive functions at age 70 , but these effects are almost nullified after adjusting for intelligence test scores at age 11 . With regard to structural brain variables, less relative decline in cognitive function between age 11 and the $70 \mathrm{~s}$ is associated with better white matter structure [30], having fewer white matter hyperintensities [7], having less brain atrophy [31] and having fewer iron deposits [32]. Even with brain imaging-cognition associations there 
is some confounding; for example, the cross-sectional association between cognitive function in older age and brain cortical thickness was almost wholly accounted for by cognitive ability test scores at age 11 years [33].

Given the wealth of data available in the LBC1936 study, our aim is to extend post-mortem characterization of each donated brain to the level of the synapse, to document synaptic alterations as a potential substrate for the neuropathological underpinnings of cognitive change during ageing. Until recently it was not possible to accurately quantify synaptic and axonal structure and protein composition in human post-mortem brain sections. However, here we describe a modified tissue processing procedure at autopsy, which allows the preservation of human brain ultrastructure for super-resolution electron microscopy and array tomography. We have pioneered the use of this technique for human tissue and published a detailed protocol to allow others to utilize this approach [34], however until now this technique has never been used to study nonpathological cognitive ageing. This approach will allow detailed analysis of human brain at the level of individual synapses and assessment of numerous proteins (pathological or physiological) at these synapses. Ultimately, this will allow us for the first time to characterize, within an individual, youth intelligence to older age-related cognitive change and the underlying macroscopic and microscopic alterations within the cells and synapses of the brain at post-mortem.

In summary, here we present methods and pilot data from the first donated LBC1936 brain tissue and describe in detail the multi-disciplinary post-mortem approach to be used for the remaining cohort, which we expect will ultimately generate a wealth of information on brain health and underlying pathology.

\section{Materials and methods Cognitive testing}

When recruited into the study at age 70 years, all LBC1936 participants underwent a range of cognitive tests covering reasoning, memory, executive function and speed of information processing, as described previously [35]. Included in the range of tests, was the same Moray House Test No.12 they took at the age of 11 . This is a well-validated test of general intelligence.

\section{MRI acquisition and processing}

All MRI data were acquired using a GE Signa Horizon HDxt 1.5 T clinical scanner (General Electric, Milwaukee, WI) equipped with a self-shielding gradient set $(33 \mathrm{mT} / \mathrm{m}$ maximum gradient strength) and manufacturer supplied eight-channel phased-array head coil. As described in detail in [36] the examination comprised the following wholebrain structural sequences acquired with contiguous slice locations: T2- (T2W), T2*- (T2*W) and FLAIR-weighted axial scans (80, 80 and 40 slices respectively) and a highresolution T1-weighted (T1W) volume sequence acquired in the coronal plane (160 slices). The field-of-view was $256 \times 256 \mathrm{~mm}$ in all cases, with voxel dimensions of $1 \times$ $1 \times 2 \mathrm{~mm}$ for T2W and T2*W, $1 \times 1 \times 4 \mathrm{~mm}$ for FLAIR, and $1 \times 1 \times 1.3 \mathrm{~mm}$ for T1W. These data were then converted from DICOM (http://dicom.nema.org) to NIfTI-1 (http://nifti.nimh.nih.gov/nifti-1) format and registered together using FSL tools (http://www.fmrib.ox.ac.uk/fsl) to allow visualization.

\section{Post-mortem analysis}

Use of human tissue for post-mortem studies has been reviewed and approved by the Edinburgh Brain Bank ethics committee and the ACCORD medical research ethics committee, AMREC (ACCORD is the Academic and Clinical Central Office for Research and Development, a joint office of the University of Edinburgh and NHS Lothian). The Edinburgh Brain Bank is a Medical Research Council funded facility with research ethics committee (REC) approval (11/ES/0022). Tissue from three donors was used for this study and their details are found in Table 1.

Table 1 Post-mortem details of the tissue donors in this study

\begin{tabular}{llll}
\hline & LBC & AD & MND \\
\hline Sex & Female & Male & Male \\
Age & 77 & 57 & 50 \\
PM Delay & $75 \mathrm{~h}$ & $58 \mathrm{~h}$ & $89 \mathrm{~h}$ \\
Brain Weight (g) & 1320 & 1200 & 1350 \\
Brain pH & 6.5 & 5.9 & 6.5 \\
Clinical Notes & Small Vessel Disease & Alzheimer's Disease & Motor Neuron Disease \\
& Braak I & Braak VI & \\
& Old Microinfarcts & & \\
& Cerebral Amyloid Angiopathy (CAA) & & \\
\hline
\end{tabular}




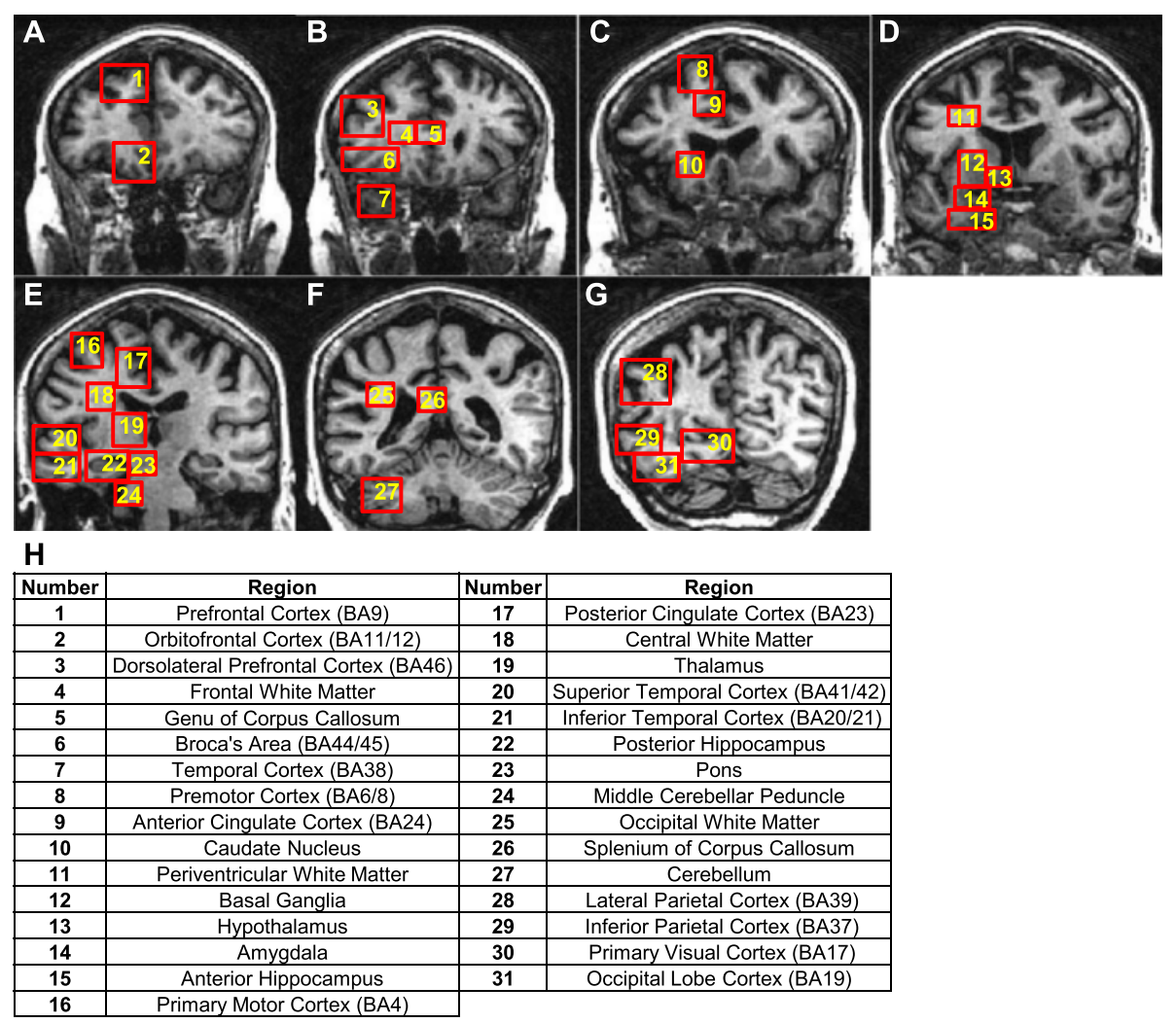

Fig. $1 \mathrm{MRI}$ showing the location of brain regions sampled for post-mortem analysis. FLAIR-weighted, coronal MR images from anterior to posterior (a-g) of the LBC1936 participant's brain. Post-mortem, regions were collected for analysis as highlighted by the numbered boxes. $\mathbf{h}$ Numbered boxes correspond to the brain regions labelled in the table, with corresponding Brodmann Area for each cortical region provided

At post-mortem the brain was removed as detailed previously [37] and cut into coronal slices while still unfixed. Regions of interest were then dissected from each coronal slice. As can be seen in Fig. 1, 31 regions are marked for dissection however some of these are subdivided into other regions of interest within that block. The hippocampus is divided into Entorhinal Cortex, CA1 and Dentate Gyrus, the Basal Ganglia is separated into the Substantia Nigra, Putamen and Internal Capsule and BA6/8, BA23 and BA39 also contain underlying subcortical white matter blocks. This adds another 7 regions, bringing the total to 38. Each region of interest from one hemisphere is then processed for paraffin embedding or frozen for biochemistry. Samples from the other hemisphere are placed in small bijous containing $0.1 \mathrm{M}$ Phosphate Buffer (PB) and dissected into smaller segments. These samples are processed for array tomography [34] or electron microscopy and the remaining tissue is snap frozen on dry ice for biochemistry.

\section{Biochemistry}

Chemicals were obtained from Sigma-Aldrich UK unless otherwise noted. Synaptoneurosomes and crude homogenates were prepared according to [38]. Briefly, $200 \mathrm{mg}$ of fresh-frozen human brain tissue was homogenized in a glass dounce homogenizer with $1 \mathrm{~mL}$ ice-cold buffer A $(25 \mathrm{mmol} / \mathrm{L}$ HEPES pH 7.5, $120 \mathrm{mmol} / \mathrm{L} \mathrm{NaCl}$, $5 \mathrm{mmol} / \mathrm{L} \mathrm{KCl}, 1 \mathrm{mmol} / \mathrm{L} \mathrm{MgCl}_{2}$, and $2 \mathrm{mmol} / \mathrm{L}$ $\mathrm{CaCl}_{2}$ ), supplemented with $2 \mathrm{mmol} / \mathrm{L}$ dithiothreitol (DTT), protease inhibitors (Roche complete mini), and phosphatase inhibitors (Calbiochem \#524629). The homogenate was passed through 2 layers of $80-\mu \mathrm{m}$ nylon filters (Millipore, Watford, UK), and a 200- $\mu \mathrm{L}$ aliquot of the filtered homogenate was saved. The saved aliquot was mixed with $200 \mu \mathrm{L}$ water and $60 \mu \mathrm{L}$ $10 \%$ SDS, to prepare the crude homogenate.

To prepare synaptoneurosomes, the remainder of the homogenate was passed through a $5-\mu \mathrm{m}$ Durapor membrane filter (Millipore) to remove large organelles and nuclei and centrifuged at $1000 \mathrm{~g}$ for 5 mins. The nonsynaptic supernatant containing cytoplasmic proteins was removed, and the pellet was washed once with buffer $\mathrm{A}$ and centrifuged again, yielding the synaptoneurosome pellet. This was suspended in $400 \mu \mathrm{L}$ of Buffer B (50 mmol/L Tris [pH 7.5], $1.5 \%$ SDS, and $2 \mathrm{mmol} / \mathrm{L}$ DTT) and boiled for 5 mins. $10 \%$ SDS was added to the supernatant fraction to bring the concentration to $1.5 \% \mathrm{SDS}$ and this was boiled for $5 \mathrm{~min}$. 
Table 2 Primary antibody information

\begin{tabular}{|c|c|c|c|c|}
\hline \multicolumn{5}{|l|}{ Western blots } \\
\hline Antibody & Company & Code & Dilution & \\
\hline PHF1 & Peter Davies & & $1: 500$ & \\
\hline Tau13 & Covance & MMS-520R-500 & $1: 2000$ & \\
\hline GAPDH & Abcam, & Ab8245 & $1: 2000$ & \\
\hline Beta-actin & Abcam & Ab8226 & $1: 2000$ & \\
\hline Synaptophysin & Abcam & Ab8049 & $1: 5000$ & \\
\hline Beta-III-tubulin & Abcam & Ab18207 & $1: 1000$ & \\
\hline $\mathrm{MBP}$ & AbD Serotec & MCA409s & $1: 500$ & \\
\hline Histone & Abcam & Ab1791 & $1: 1000$ & \\
\hline VDAC1/Porin & Abcam & Ab34726 & $1: 500$ & \\
\hline GluN2B & BD Biosciences & 610416 & $1: 500$ & \\
\hline Synapsin & Millipore & AB1543P & $1: 20000$ & \\
\hline \multicolumn{5}{|l|}{ Neuropathology } \\
\hline Antibody & Company & Code & Dilution & Pre-treatment \\
\hline Beta Amyloid (BA4) & Dako & M087201-2 & 1:100 & $98 \%$ formic acid 5 min \\
\hline Alpha Synuclein & Life Technologies & $32-8100$ & $1: 200$ & Pressure cooker/formic acid \\
\hline TDP-43 & 2B Scientific & CAC-TIP-PTD-MO1 & $1: 4000$ & Pressure cooker/citric acid \\
\hline pTau (AT8) & Thermo & MN1020 & $1: 2500$ & None \\
\hline Ubiquitin & Dako & Z0458 & $1: 500$ & Pressure cooker/citric acid \\
\hline GFAP & Dako & Z0334 & $1: 800$ & None \\
\hline CD68 & Dako & M0876 & $1: 100$ & Pressure cooker/citric acid \\
\hline \multicolumn{5}{|l|}{ Array tomography } \\
\hline Antibody & Company & Code & Dilution & Secondary antibody \\
\hline AW7 & Dominic Walsh & & $1: 1000$ & Donkey a Rabbit - AF488 \\
\hline Synaptophysin & Abcam & Ab8049 & $1: 50$ & Donkey a Mouse - AF594 \\
\hline ApoE & Abcam & Ab7620 & $1: 50$ & Donkey a Goat - AF647 \\
\hline PSD95 & Abcam & Ac12093 & $1: 50$ & Donkey a Goat - AF488 \\
\hline
\end{tabular}

Protein levels were analyzed in each sample using a BCA protein assay (Thermo Fisher Scientific, Loughborough, UK). $5 \mu \mathrm{g}$ of protein was loaded into precast NuPAGE 4$12 \%$ Bis-Tris polyacrylamide 15 well gels (Invitrogen, Paisley, UK) alongside a molecular weight marker (Li-Cor, Cambridge, UK). Electrophoresis was performed at $100 \mathrm{~V}$ for $2 \mathrm{~h}$. Proteins were electro-transferred to nitrocellulose membrane (Bio-Rad, Hemel Hempstead, UK) at $30 \mathrm{~V}$ for $1.5 \mathrm{~h}$ using the XCell $\mathrm{II}^{\mathrm{mM}}$ Blot Module system (Invitrogen, Paisley, UK) in tris-glycine transfer buffer. Membranes were incubated in $10 \mathrm{ml}$ Odyssey blocking buffer (Li-Cor, Cambridge, UK) diluted 1:1 with Phosphate Buffered Saline (PBS) for $1 \mathrm{~h}$ and then incubated with primary antibodies overnight. Primary antibodies used for western blots are shown in Table 2. After washing six times for $5 \mathrm{~min}$ in PBS/0.1 \% Tween-20 solution, the membranes were incubated for $1 \mathrm{~h}$ with the appropriate 680 and 800 IR dye secondary antibodies (1:50000, LI-COR Biosciences). The membranes were washed again in PBS/0.1 \% Tween-20 solution. The membranes were imaged using an Odyssey infrared imaging system, corrected for background, and analyzed using Odyssey software (LI-COR Biosciences). For figure preparation the imaged blots were cut to only show the bands that were used for the densitometry analysis. Molecular weights are provided in the figure legends.

\section{Neuropathology}

Fresh post-mortem tissue blocks (approximately $1 \mathrm{~cm}^{3}$ ) from our regions of interest were fixed in $10 \%$ formalin for a minimum of $24 \mathrm{~h}$. Tissue was then dehydrated in an ascending series of alcohol (70-100\%), followed by three xylene washes, all for $4 \mathrm{~h}$ each. Next, three paraffin waxing stages ( $5 \mathrm{~h}$ each) were performed to ensure full penetration of the embedding wax and finally these blocks were allowed to cool. Tissue sections were cut on a Leica microtome at 
$13 \mu \mathrm{m}$ and collected on glass slides. All sections were dried at $40{ }^{\circ} \mathrm{C}$ for at least $24 \mathrm{~h}$ before staining.

Immunohistochemistry was performed using standard protocols, enhanced using the Novolink Polymer detection system and visualized using 3,3'-Diaminobenzidine (DAB) as chromogen. See Table 2 for antibody information. Slides were finally counterstained with hematoxylin for $30 \mathrm{~s}$ to stain cell nuclei. Images were scored semi-quantitatively according to the estimated level of pathology. Additional file 1: Figure S1 contains example images for each stain and pathology score.

\section{Burden quantification}

All sections stained for $\beta$-amyloid and GFAP were assessed for plaque and astrocytic burden respectively using Stereo Investigator. Cortical grey matter was outlined in each section and immune-positive objects identified using an automated colour-based thresholding algorithm. The area of immuno-positive cortex was expressed as a percentage of total cortex in each brain region.

\section{Cortical thickness}

Hematoxylin and eosin stained cortical sections were used to measure cortical thickness. This stain provides a clear demarcation between grey and white matter. Grey matter thickness was calculated at eight randomly selected points throughout each section. Thickness was measured from the pial surface to the border of grey/white matter, below cortical layer 6. All eight measurements were averaged to give a cortical thickness for each brain region.

\section{Neuron and microglia density}

Neuron and microglia densities were generated within the grey matter of each cortical section, stained with CD68. Using a stereological optical dissector approach (described in [39], approximately 250 fields of view were randomly scattered across the cortical grey matter. Within each field of view a dissector grid of $200 \mu \mathrm{m} \times 200 \mu \mathrm{m}$ was applied and all CD68-positive cells within the grid and touching the acceptable boundaries were counted. Neurons were identified based on the presence of large oblong nuclei and were counted within each dissector grid. Total cell counts within all grids were divided by total imaged volume to generate densities of cells per $\mathrm{mm}^{3}$. Finally, CD68-positive cell soma cross-sectional area was measured to ensure changes in cell size were not influencing our total counts. We discovered no change in CD68-positive cell size between cases when more than 300 cells per case from two cortical regions were analysed (data not shown).

\section{Electron microscopy}

Brain samples were prepared for electron microscopy as previously outlined in detail [34]. Briefly, fresh postmortem samples, stored in $0.1 \mathrm{M} \mathrm{PB}$ were trimmed into small cortical blocks and fixed in $4 \%$ paraformaldehyde and $2.5 \%$ glutaraldehyde in $0.1 \mathrm{M} \mathrm{PB}$ for $48 \mathrm{~h}$. Blocks were washed twice in $0.1 \mathrm{M} \mathrm{PB}$, then cut at $70 \mu \mathrm{m}$ with a vibratome. Sections were treated with osmium tetroxide (1\% in $0.1 \mathrm{M} \mathrm{PB}$ ) for 30 mins (protected from light) and dehydrated in an ascending series of ethanol and propylene oxide, before embedding in Durcupan resin. During dehydration the sections were treated with uranyl acetate ( $1 \%$ in $70 \%$ ethanol) for $40 \mathrm{mins}$ in the dark. Durcupan resin was polymerized for $48 \mathrm{~h}$ at $56^{\circ} \mathrm{C}$. Small regions of interest were cut from the Durcupan-embedded sections and glued onto Durcupan blocks, before cutting $70 \mathrm{~nm}$ ribbons with an ultracut microtome (Leica) using a Jumbo Histo Diamond Knife (Diatome, Hatfield, PA). Ribbons were collected on grids and stained with lead citrate before imaging on a JEOL JEM-1011 transmission electron microscope (TEM) with Hamamatsu ORCA digital camera. For synapse analysis, an average of 16 images per region (range 10-28) were taken at 20,000x magnification in a systematic, random fashion from blocks from BA17, BA24, BA41/42, BA44/45, BA46, BA6/8, BA9, entorhinal cortex, and $C A 1$ stratum radiatum from the LBC1936 and AD cases.

For white matter analysis, an average of 5 images at $8,000 \times$ magnification were taken in a systematic random fashion from 4 white matter regions of the LBC1936 case (BA39 subcortical white matter, BA6/8 subcortical white matter, genu of the corpus callosum, and periventricular white matter). G-ratios were measured as described previously [40]. TEM images were coded for blind analysis. In grey matter images, synapses were defined by the presence of at least 3 synaptic vesicles in the presynaptic terminal and a clear postsynaptic density. For each synapse, the following measurements were made: apposition length, number of presynaptic vesicles, pre and postsynaptic terminal cross-sectional areas. Each synapse was also classified as excitatory or inhibitory (based on intensity of PSD, synaptic vesicle morphology, and cleft width) and the presence of multivesicular bodies (MVB), mitochondria, spine apparatus (in the postsynapse), perforated postsynaptic density (PSD), and dark degenerating terminals was noted. In white matter images, diameters of axons and axon + myelin sheaths were measured and the presence of mitochondria was noted. A total of 1600 axons were measured.

\section{Array tomography}

Brain samples were prepared for array tomography as previously outlined in detail [34]. A summary flowchart of the steps involved is provided in Additional file 2: Figure S2. Briefly, fresh post-mortem samples were trimmed into small cortical blocks and fixed in $4 \%$ paraformaldehyde for $3 \mathrm{~h}$. Samples were dehydrated through ascending ethanol washes and embedded in LR White resin. Tissue blocks 
were cut into ribbons of $70 \mathrm{~nm}$ sections and collected on coverslips. Ribbons were immunostained on "day1" with antibodies against Synaptophysin, AW7 and ApoE overnight (see Table 2 for antibody details). Sections were then developed with fluorescently-labelled secondary antibodies and images obtained along the ribbon using a Zeiss axio Imager Z2 epifluorescent microscope equipped with a CoolSnap digital camera and AxioImager software with array tomography macros (Carl Zeiss, Ltd, Cambridge UK). High-resolution images were obtained with a $63 \times 1.4$ NA Plan Apochromat objective. Coverslips were washed twice in TBS and stripped of all antibodies with a $10 \mathrm{~min}$ wash in stripping buffer $(0.2 \mathrm{M} \mathrm{NaOH}$ and $0.02 \%$ SDS in TBS). The staining protocol was then repeated ("day2") with antibodies against PSD-95 (see Table 2 for antibody details). Negative controls lacking primary antibody were run alongside to ensure the specificity of the protocol. These were always blank (data not shown). Images from "day1" and "day2" were combined, aligned, and regions of interest (crops) chosen in the neuropil. These crops were coded for blind analysis and thresholded with automated algorithms in ImageJ. Synaptic puncta counts and volumes were generated using our own MATLAB scripts and analysis of co-localization with pathological proteins expressed as a percentage of total synapses. All ImageJ macros and MATLAB scripts will be made available upon request.

\section{Statistics}

GraphPad Prism was used to test array tomography data for normality (Kolmogorov-Smirnov test), following which parametric ( $t$-test or ANOVA) or non-parametric (Kruskal-Wallis or Mann-Whitney) tests were performed as required. SPSS was used to compute statistics for electron microscopy data. Normality of data was tested with a Shapiro-Wilkes test. Normally distributed data were analyzed with parametric statistics and non-normal data with non-parametric tests as required. Significance was reported when $P<0.05$.

\section{Results}

\section{Cognitive summary of the first LBC1936 brain donor}

The LBC1936 participant scored either 28 or 29 out of 30 on the Mini-Mental State Examination on three occasions of testing at about 70,73 , and 76 years, indicative of no substantial cognitive decline. The participant's scores on the Moray House Test (verbal reasoning; age 11, 70 and 76), Wechsler Logical Memory (verbal declarative memory; age 70, 73, and 76), and Wechsler Digit Symbol (processing speed; age 70, 73 and 76) were between 0.5 and 1.0 standard deviations (SD) higher than their LBC1936 contemporaries at age 11 or 70 , but only between 0 and 0.5 SD higher at age 76. On Wechsler Matrix Reasoning (non-verbal reasoning; ages 70, 73, and 76) the participant's score was consistently about 0.25 SD below the
LBC1936 cohort mean. In summary, the participant exhibited no cognitive decline and generally scored above the average LBC1936 score in most tests, with some decline in that advantage at the last round of tests. At the third test, the participant mentioned suffering a small stroke since the second wave (aged 73) and that following the stroke she had noticed minor memory problems. The participant died 8 months after the final cognitive test.

\section{Structural MRI}

To illustrate how the degree of cortical thinning and ventricular enlargement present in our subject compared with that seen across the entire LBC1936, Fig. 2a shows representative coronal images from twelve participants, arranged by degree of cortical thinning and ventricular enlargement from top left to bottom right. Cortical thinning is a common feature of brain ageing [41] and in Fig. 2c it can be seen that the LBC1936 case here also exhibits some localized cortical thinning, highlighted by red arrows. The level of cortical thinning was described as being within normal limits for age, by the study's radiologist. White matter hyperintensities are a common feature of brain ageing and are more common in patients with cardiovascular problems and neurodegenerative diseases such as Alzheimer's [42]. They tend to be found in the deep white matter tracts of the brain [42] and as can be seen in Fig. 2b, their presence varies strikingly between the age-matched, non-demented LBC1936 cohort participants. The LBC1936 participant in this study exhibited a number of mild periventricular and subcortical white matter hyperintensities as highlighted by the blue arrows in Fig. 2d. No microhaemorrhage or mineralization was noted in the T2-weighted (Fig. 2e) or GRE-weighted scans (Fig. 2f). In fact, the only other feature of note from the imaging studies was the remains of small old infarcts in the left frontal and lateral occipital lobes.

\section{Outline of post-mortem studies}

Post-mortem analysis can be performed on up to 38 freshly dissected brain regions (Fig. 3). Once removed, these fresh tissue blocks will be processed in numerous ways to allow comprehensive characterization, from gross biochemistry to super-resolution electron microscopy.

\section{Neuropathology}

The neuropathological correlates of cognitive decline in healthy ageing (if any) are not well understood. All brain regions were immuno-stained with antibodies against proteins known to be involved in neurodegenerative diseases associated with cognitive decline. Semi-quantitative scoring of the immunostaining revealed a very low level of pathology in the non-demented LBC1936 brain compared to the AD case (Table 3). Braak scoring revealed the LBC1936 had mild early stage tau pathology (Braak stage I), whereas 


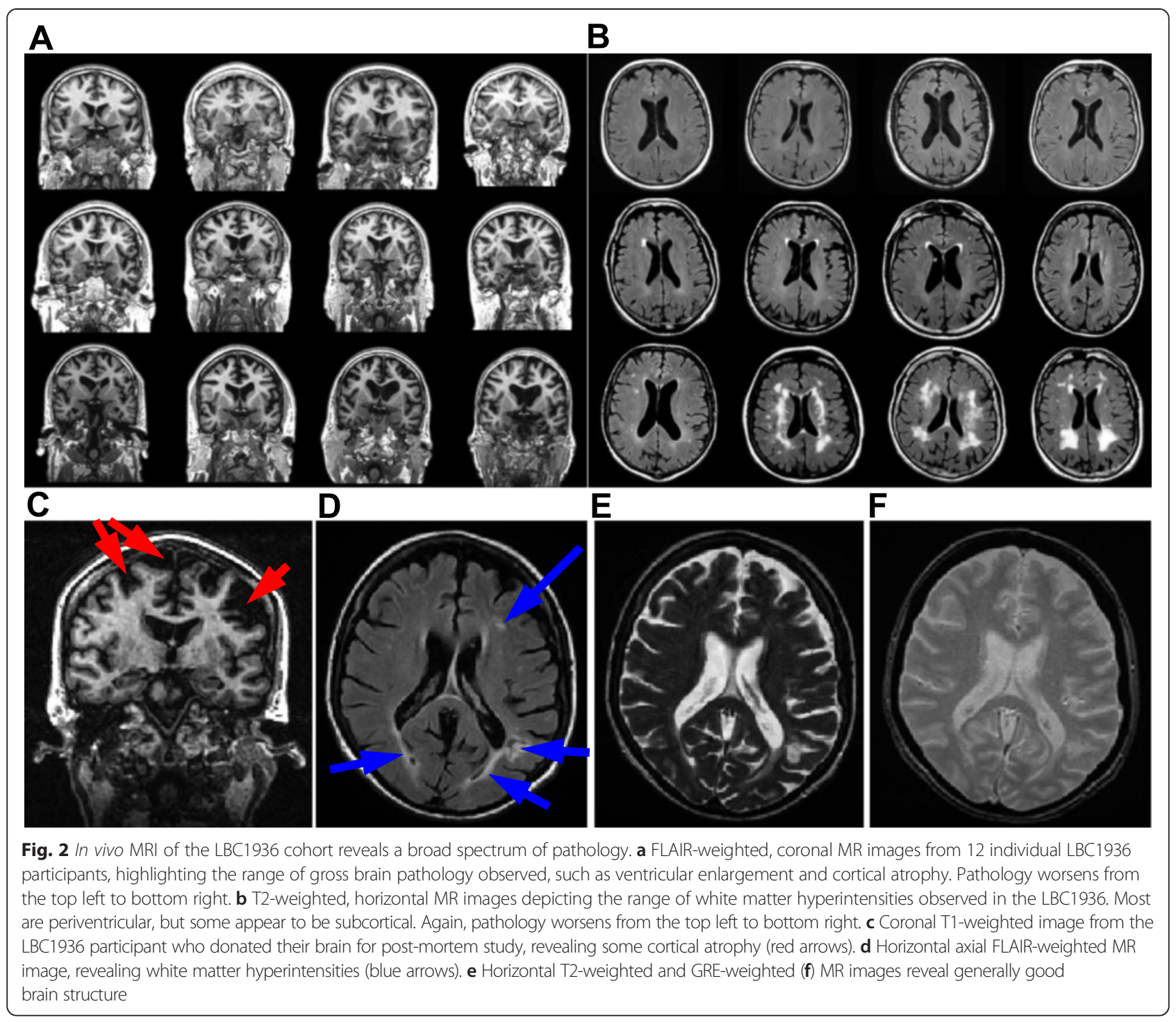

the AD case had much more severe pathology (Braak stage VI). To allow more meaningful association between cognitive status and the extent of pathology in the brain of our LBC1936 subjects, we used stereological approaches to quantify some of the pathological stains. The two major pathological hallmarks of $\mathrm{AD}$ are extracellular $\beta$-amyloid plaques and intracellular tau-positive neurofibrillary tangles. However it is known that these can also appear in cognitively normal aged brains [43, 44]. The LBC1936 brain contained a very small number of amyloid plaques, mostly restricted to the entorhinal cortex (Fig. $4 \mathrm{a}, \mathrm{e}$ ). Figure $4 \mathrm{a}$ shows the premotor cortex (BA6/8) devoid of cortical amyloid plaques, yet quite distinct amyloid staining of some blood vessels within and on the surface of the cortex (Fig. 4a insert), revealing that the participant suffered from cerebral amyloid angiopathy (CAA). This vascular amyloid deposit is found in approximately $50 \%$ of people over the age of 70 years and is not dependent on more global Alzheimer's-like deposition throughout the grey matter [45]. This was in striking contrast to the AD brain, which contained a high plaque burden throughout the premotor cortex (Fig. 4c) and more globally throughout the brain (Fig. 4e). Interestingly, despite this massive amyloid burden, the blood vessels appeared mostly free of CAA (Fig. 4c insert).

The other pathological hallmark of Alzheimer's disease is tau pathology and in the hippocampal CA1 region from the LBC1936 brain, only a few rare tau-positive neurites (Fig. 5c) were found. As expected, the AD hippocampus was full of tau-positive tangles within neuronal somata and dystrophic neurites (Fig. 5d).

Glia are integral for normal brain function and are found throughout the brain under normal conditions. However, gliosis is a common feature of many neuropathologies [46] and as shown in Fig. 4b, d, f the astrocytic burden in the AD brain was higher than the LBC1936 brain in all regions 


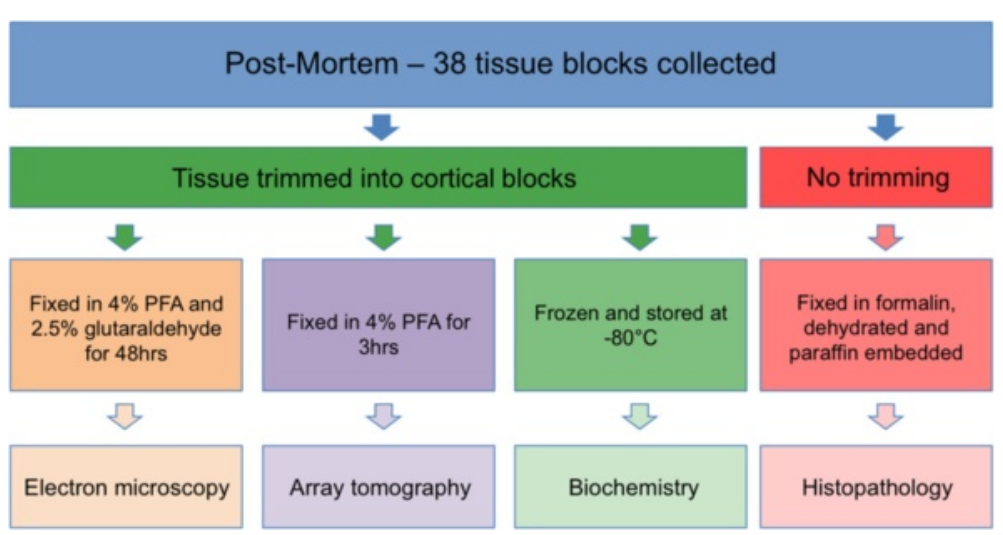

Fig. 3 Summary of post-mortem tissue processing. Flowchart showing the numerous processing techniques employed, allowing us to gain cellular and subcellular information on brain integrity

analyzed. Interestingly, microglial densities were much more variable between cases with higher densities found in some cortical regions of the LBC1936 brain (Fig. 5a, b, k). Transactive response DNA binding protein $43 \mathrm{kDa}$ (TDP-43) is a transcriptional repressor protein linked to fronto-temporal dementia (FTD) and Amyotrophic Lateral Sclerosis (ALS) [47]. TDP-43 is normally found in the neuronal nucleus but in patients with FTD it spreads into the cytoplasm in a phosphorylated and ubiquitinated form [47]. The staining of TDP-43 in the LBC1936 hippocampus appeared to be mostly nuclear (Fig. 5e), and this pattern was similar in all other regions (Table 3), however in some cortical regions, translocation of TDP-43 was apparent in a small population of neurons as the strong nuclear labeling was replaced with diffuse somatic labeling. The AD brain exhibited dense cytoplasmic staining and a complete clearing of nuclear staining in a large subset of neurons in many cortical regions (Table 3 ) and the hippocampal CA1 region (Fig. 5f). Ubiquitin is a small regulatory protein that plays an important role in the post-translational modification of numerous proteins, the addition of which often leads to protein aggregation or degradation [48]. There was a striking difference in the level and pattern of ubiquitin staining between both cases with mostly glial staining in the LBC1936 hippocampus (Fig. 5g) and very strong somatic labelling of many neurons in the AD hippocampus (Fig. 5h). This pattern was found throughout the brain regions analysed (Table 3). Alpha-synuclein is highly expressed in the brain and is an integral protein of the presynaptic terminal. Insoluble fibrils are often found in neuronal somata in diseases such as multiple-system atrophy, Parkinson's disease, Alzheimer's disease and dementia with Lewy bodies [49]. However, no distinct alphasynuclein aggregates were found in either the LBC1936 (Fig. 5i) or the AD hippocampus (Fig. 5j). This pattern was similar throughout the brain of each case
(Table 3). Semi-quantitative scoring of all stains in all regions analyzed is summarized in Table 3.

As Alzheimer's disease progresses, neurons die and brain atrophy occurs. This is highlighted in our neuron density counts, revealing a generally higher neuronal density in most regions of the LBC1936 brain (Fig. 5l). Neuron density values can be influenced by cortical thickness, as the packing of cells can increase in smaller volumes. In most regions, the cortical thickness was similar between cases, however in EC and BA46 the AD cortex appears thicker, but thinner than the LBC1936 cortex in BA41/42 (Fig. 5m).

In summary, the neuropathological assessment of the LBC1936 brain suggests good structural integrity although the brain does show a mild neuropathological burden, which maybe suggestive of early neurodegenerative processes.

\section{Biochemistry}

To assess the levels of synaptic proteins, blots of crude homogenate were stained for the pre-synaptic marker synaptophysin. Synaptophysin staining revealed a decrease in synapses in most regions of the $\mathrm{AD}$ brain compared to the LBC1936 (Fig. 6a), except the EC and hippocampus (Fig. 6a). GFAP staining revealed a group of bands due to the expression of multiple GFAP isoforms in human brain [50]. In many cortical regions the $\mathrm{AD}$ samples expressed higher levels of GFAP than the LBC1936 case (Fig. 6b) as expected from our stereological burden counts (Fig. 4), however in BA46 and the hippocampus the levels were higher in the LBC1936 sample. Tau blots revealed a higher level of this neuronal protein in all regions of the LBC1936 brain compared to $\mathrm{AD}$ (Fig. 6c), possibly due to neuronal loss observed in advanced AD [51]. Strikingly, the phosphorylated form of tau was present in all regions of the AD brain, but virtually absent in the LBC1936 samples (Fig. 6d). Collectively, these blots highlight low levels of AD-like 
Table 3 Semi-quantitative scoring of neuropathological markers

\begin{tabular}{|c|c|c|c|c|c|c|c|}
\hline \multirow{3}{*}{$\begin{array}{l}\text { Region } \\
\text { BA9 }\end{array}$} & \multirow{3}{*}{$\begin{array}{l}\text { Stain } \\
\text { TDP43 }\end{array}$} & \multirow{2}{*}{\multicolumn{2}{|c|}{$\frac{\mathrm{LBC} \quad \mathrm{AD}}{\text { Score }}$}} & \multirow{3}{*}{$\begin{array}{l}\text { Region } \\
\text { BA41/42 }\end{array}$} & \multirow{3}{*}{$\begin{array}{l}\text { Stain } \\
\text { TDP43 }\end{array}$} & LBC & $A D$ \\
\hline & & & & & & \multicolumn{2}{|c|}{ Score } \\
\hline & & - & ++ & & & - & ++ \\
\hline & pTAU & - & +++ & & PTAU & - & +++ \\
\hline & BA4 & + & +++ & & BA4 & + & +++ \\
\hline & a-Syn & - & - & & a-Syn & - & - \\
\hline & GFAP & + & +++ & & GFAP & ++ & +++ \\
\hline & CD68 & + & +++ & & CD68 & + & + \\
\hline & UBIQ & + & +++ & & UBIQ & + & +++ \\
\hline \multirow[t]{7}{*}{ BA44/45 } & TDP43 & + & + & EC & TDP43 & + & ++ \\
\hline & pTAU & - & +++ & & pTAU & + & +++ \\
\hline & BA4 & - & +++ & & BA4 & ++ & +++ \\
\hline & a-Syn & - & - & & a-Syn & - & - \\
\hline & GFAP & + & ++ & & GFAP & + & +++ \\
\hline & CD68 & ++ & ++ & & CD68 & + & ++ \\
\hline & UBIQ & + & +++ & & UBIQ & ++ & +++ \\
\hline \multirow[t]{7}{*}{ BA46 } & TDP43 & + & ++ & BA17 & TDP43 & + & ++ \\
\hline & PTAU & - & +++ & & PTAU & - & +++ \\
\hline & BA4 & - & +++ & & BA4 & + & +++ \\
\hline & a-Syn & - & - & & a-Syn & - & - \\
\hline & GFAP & + & ++ & & GFAP & + & +++ \\
\hline & CD68 & + & ++ & & CD68 & + & + \\
\hline & UBIQ & + & +++ & & UBIQ & + & +++ \\
\hline \multirow[t]{7}{*}{ BA6/8 } & TDP43 & + & ++ & BA24 & TDP43 & + & ++ \\
\hline & PTAU & - & +++ & & pTAU & - & +++ \\
\hline & BA4 & + & +++ & & BA4 & - & +++ \\
\hline & a-Syn & - & - & & a-Syn & - & - \\
\hline & GFAP & + & +++ & & GFAP & + & ++ \\
\hline & CD68 & + & +++ & & CD68 & + & ++ \\
\hline & UBIQ & + & +++ & & UBIQ & + & +++ \\
\hline
\end{tabular}

BA9 $=$ Prefrontal cortex, $\mathrm{BA} 44 / 45=$ Broca's area, $\mathrm{BA} 46=$ Dorsolateral Prefrontal cortex, BA6/8 = Premotor cortex, BA41/42 = Superior Temporal cortex, $\mathrm{EC}=$ Entorhinal cortex, BA17 $=$ Primary Visual cortex, BA24 $=$ Anterior Cingulate cortex. " $-"$ " = no pathology, " + " = mild pathology, $"++"=$ moderate pathology, " $+++"=$ strong pathology. Example images for each score are found in Additional file 1: Figure S2

pathology in the non-demented LBC1936 brain, however whole cell homogenates can only provide an estimate of synaptic proteins. Many non-synaptic proteins and synaptic proteins that have not yet trafficked to the synapse are present in whole cell homogenates, providing an inaccurate measure of localized synaptic proteins. To provide more accurate synaptic measurements, synaptoneurosome preparations were generated to isolate the synaptic components from the whole homogenate (Fig. 7a). To assess the quality of our preparations we used a recently established method for analysing synaptic protein integrity in brain homogenates [52]. This revealed all our samples except the LBC1936 EC contained good protein integrity (Fig. 7b).
These new preparations reveal a more subtle change (if any) in synaptophysin levels in the remaining synapses between the LBC1936 and AD samples (Fig. 7c). Total tau blots revealed the presence of this protein at synapses and showed that again in most regions, similar levels were observed in the LBC1936 samples compared to AD synaptoneurosomes (Fig. 7d). Toxic forms of tau are thought to accumulate in axon terminals in $\mathrm{AD}[38,53]$ and our blots reveal significant accumulation of phosphorylated tau in the AD synaptoneurosomes and none in the LBC1936 samples (Fig. 7e). Synapses are metabolically demanding structures, requiring mitochondria in close proximity to maintain physiological function. Staining our synaptoneurosomes for the mitochondrial marker VDAC1 revealed the presence of synaptic mitochondria in all samples, with similar levels in most samples (Fig. 7f). However, strikingly higher levels were found in LBC1936 BA41/42 and AD EC compared to their matching sample (Fig. 7f).

The in vivo MRI scans from the LBC1936 participant revealed potential underlying white matter pathology (Fig. 2). Crude homogenates of white matter regions from the LBC1936 and a motor neuron disease (MND) brain were tested for axonal tract integrity by staining for structural proteins. Axonal tubulin and tau levels were similar between cases (Fig. 8a, b), however in a number of regions myelin basic protein (MBP) levels were markedly variable (Fig. 8c). Mitochondrial levels varied dramatically between white matter regions, with most expressing low levels of VDAC1 (Fig. 8d), however in the BA39 and BA23 subcortical white matter samples the LBC1936 expressed markedly higher levels than the MND samples (Fig. 8d).

In summary, the western blots reveal generally good synaptic and axonal integrity with low levels of Alzheimer's pathology in the LBC1936 brain homogenates, in line with our observations from the immunohistochemical staining.

\section{Array tomography}

Synapses from our regions of interest were labelled preand postsynaptically with synaptophysin and PSD95 (Fig. 9a, b) and the presence of two further proteins of interest ( $\beta$-amyloid and ApoE) assessed. In total, 1500 images were analyzed, amounting to almost 162, 000 synapses. Synapses were typically observed as "snowmen" with PSD95 and synaptophysin puncta found directly opposed (see white circles in Fig. 9a, b). PSD95 densities were quite similar in most of the regions analyzed between cases, however densities in BA24 and BA41/42 appear higher in the LBC1936 case compared to the AD brain (Fig. 9c). The volume of PSD-positive compartments varied slightly between regions yet globally appeared larger in the LBC1936 brain (Fig. 9d). The variability in synaptophysin density between regions and cases, largely matched the PSD95 values, with higher densities found in BA24, BA41/ 42 and BA46 in the LBC1936 brain (Fig. 9e). 

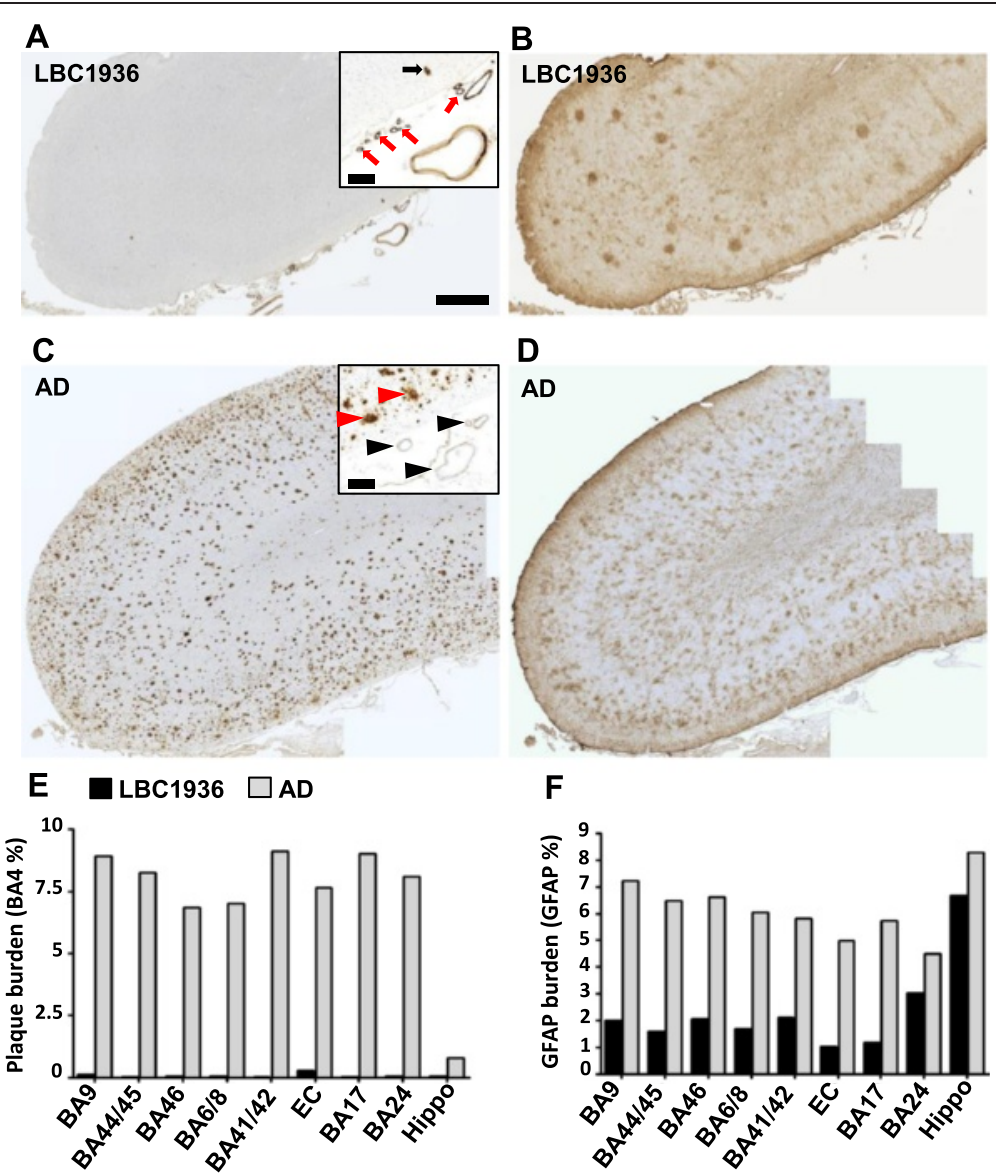

Fig. 4 Stereological assessments of amyloid plaque burden and gliosis. a Premotor cortex (BA6/8) from the LBC1936 brain contains no amyloid plaques within the cortical tissue. However, some blood vessels within the cortex (insert, black arrow) and around the pial surface (insert, red arrows) exhibit strong amyloid labelling. b LBC1936 BA6/8 region exhibits a dense network of GFAP staining in layer 1 of the cortex, with small patches of GFAP-positive cells scattered through the other cortical layers. c BA6/8 from the AD case contains a very high amyloid burden throughout the cortex. Despite the presence of cortical plaques (insert, red arrowheads) the pial vessels were largely devoid of labelling (insert, black arrowheads). $\mathbf{d}$ GFAP-positive cells were found throughout the AD BA6/8 cortical region and often found around plaque-like structures. Large scale bars $=1 \mathrm{~mm}$, insert scale bars $=0.2 \mathrm{~mm}$. e Histogram showing amyloid burden in eight cortical regions and the hippocampus in the LBC1936 case (black bars) and the AD case (grey bars). $\mathbf{f}$ Histogram showing GFAP burdens in eight cortical regions and the hippocampus from the LBC1936 and AD cases

Synaptophysin puncta volumes were larger in the LBC1936 BA24, BA46 and EC regions, but of similar size in BA17 and BA41/42 (Fig. 9f). To assess whether the synaptotoxic proteins $\beta$-amyloid and ApoE are found in synapses of the cognitively normal LBC1936 case, 3D colocalization analysis was performed (Figs. 10 and 11). A very rare population of synapses in the LBC1936 brain $(<3 \%)$ contained $\beta$-amyloid either pre- or post-synaptically (Figs. 10a and 11a,d), with a higher percentage of $\mathrm{AD}$ synapses ( $0.6 \%$ in BA24 to $5.1 \%$ in BA17) positive for $\beta$ amyloid (Figs. 10b and 11a, d). ApoE has been described in human synapses previously by our group [54] and that finding is confirmed here. In the LBC1936 case, the percentage of ApoE-positive synapses ranged from approximately $3 \%$ in BA24, to $40 \%$ in BA41/42 (Figs. 10c and 11b,e). Overall, the percentages of ApoE-positive synapses were higher and much less variable in the $\mathrm{AD}$ brain, ranging from approximately 33 to $50 \%$ (Figs. 10d and 11b, e). Interestingly, there was a very rare population of synapses that contained both of these synaptotoxic proteins. Levels were very low in the LBC1936 brain ranging from almost $0 \%$ in BA24, to $\approx 2 \%$ in BA17 (Fig. 11c, f). Higher levels were observed in the AD brain, ranging from $0.5 \%$ in BA24 to $4.8 \%$ in BA17 (Fig. 11c,f). Our array tomography approach can also be used to calculate synaptic volumes, which can be used to assess changes in the pre- and post-synapse with and without pathological burden (Fig. 11g, h).

Array tomography can also be used to build a 3D image of larger structures, such as axons. Therefore we also prepared periventricular white matter tissue (pvwm) for arrays and stained them with antibodies against axonal filaments (SMI-312R) and myelin basic protein (MBP). As can be seen in Fig. 12a and b, large caliber axonal fibers are evident 

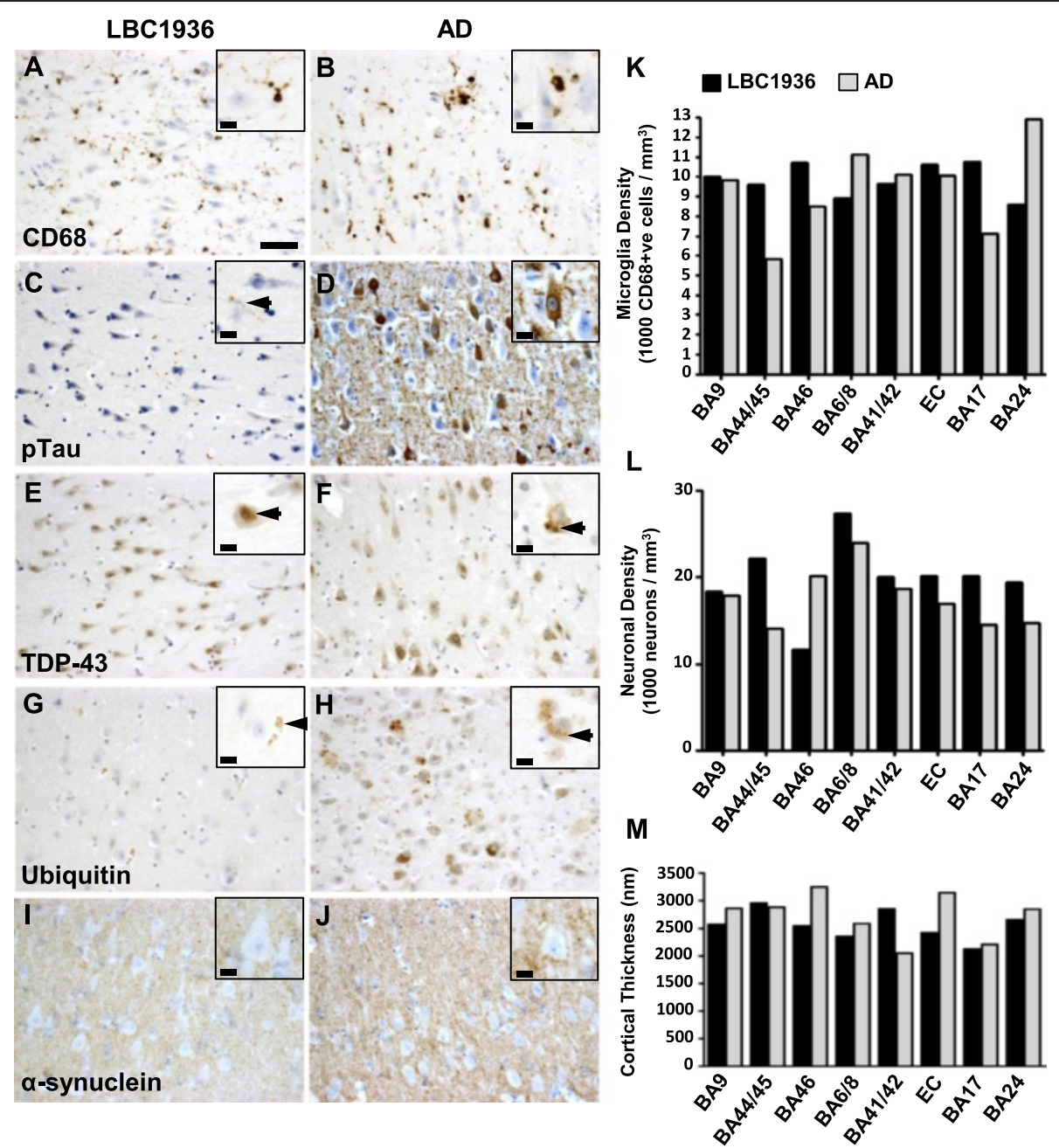

Fig. 5 Stereological assessments of brain pathology and integrity. Representative images of microglial (CD68; $\mathbf{a}+\mathbf{b})$, phosphorylated-tau $(\mathbf{c}+\mathbf{d})$, TDP-43 $(\mathbf{e}+\mathbf{f})$, ubiquitin $(\mathbf{g}+\mathbf{h})$ and $\mathbf{a}$-synuclein $(\mathbf{i}+\mathbf{j})$ staining in the hippocampus (CA1) from LBC1936 (a, $\mathbf{c}, \mathbf{e}, \mathbf{g}, \mathbf{i})$ and AD (b, $\mathbf{d}, \mathbf{f}, \mathbf{h}, \mathbf{j})$ brains. Large scale bar $=50 \mu \mathrm{m}$, insert scale bars $=10 \mu \mathrm{m}$. $\mathbf{k}$ Histogram showing neuronal densities in eight cortical regions from the LBC1936 (black bars) and AD (grey bars) brains. I Histogram showing microglial densities (CD68-positive cells) in eight cortical regions from the LBC1936 and AD brains. m Histogram of cortical thickness measurements from eight cortical regions in the LBC1936 and AD brains

in the LBC1936 and AD tissue and these are tightly wrapped in high density myelin. However, in the MND sample (Fig. 12c) the axonal fibers appear dysmorphic and loosely wrapped in low levels of myelin, when compared to the LBC1936 tissue (Fig. 12a).

In summary, our array tomography analysis provides an unprecedented wealth of detailed information on the underlying density and protein constitution of synapses in the post-mortem brain.

\section{Electron microscopy}

Images were captured from select regions in both the LBC1936 and AD brain and numerous synaptic morphological parameters were analyzed. In total, more than 350 synapses were identified as having a clear postsynaptic density and at least 3 pre-synaptic vesicles. Within individual brain regions, no differences were observed in any parameter due to the low numbers involved, however when all synapses were combined within each case, differences emerged. The LBC1936 synapses contained more presynaptic mitochondria $(\mathrm{LBC} 1936=39.7 \%, \mathrm{AD}=34.2 \%$; $\mathrm{Chi}^{2}$ test $p=0.009$ ) and most of those present appeared of normal morphology, whereas the mitochondria found in the $\mathrm{AD}$ synapses were more frequently dysmorphic $\left(\mathrm{LBC} 1936=8.3 \%, \mathrm{AD}=22.7 \%\right.$; $\mathrm{Chi}^{2}$ test $p=0.031$; Fig. 13a). Normal mitochondria generally had a clearly defined oval shape, with distinct and intact internal cristae. Dysmorphic mitochondria had fragmented internal cristae, were often electron dense and exhibited a torturous morphology. However, other pre-synaptic parameters such as vesicle number, presence of multi-vesicular bodies and electron-dense degenerating profiles, although rarely 


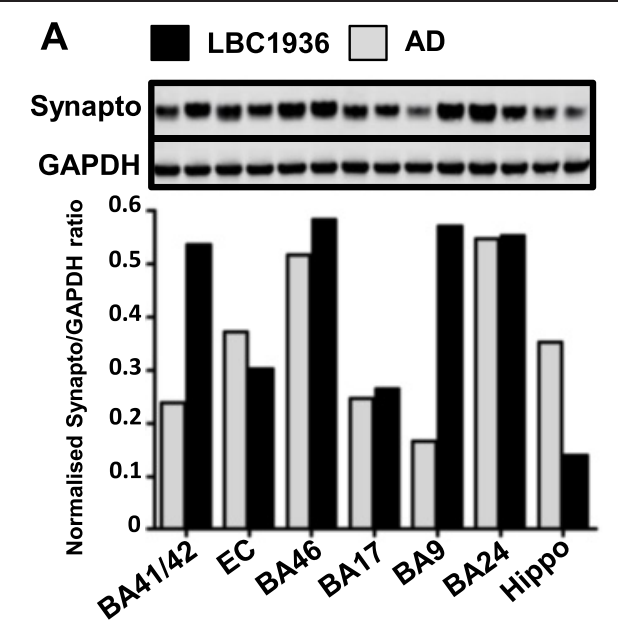

B

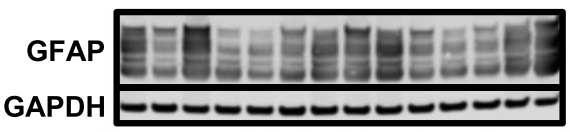

C
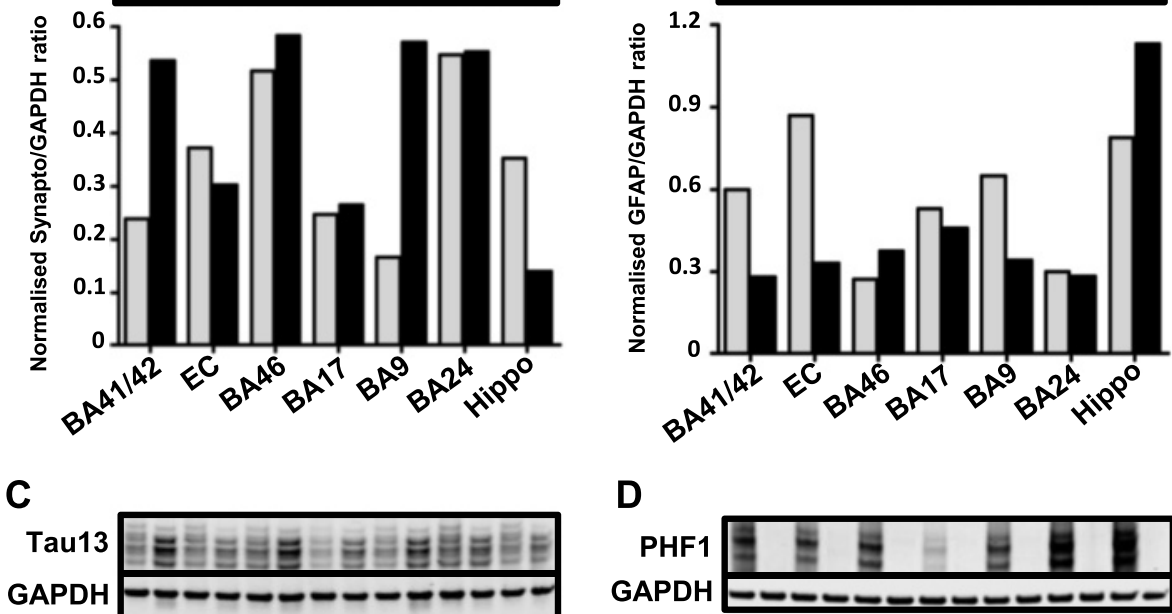

D
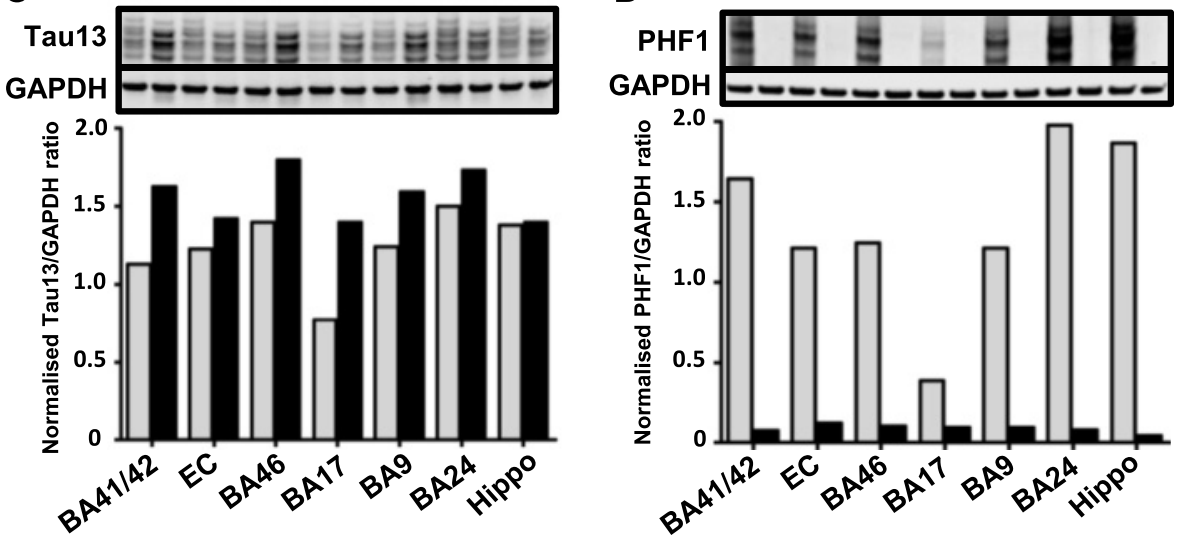

Fig. 6 High-throughput assessments of brain integrity and pathology using crude homogenate western blotting. a Representative synaptophysin (40 kDa) western blot of seven LBC1936 homogenates (black bars) and seven AD samples (grey bars). b GFAP (35-50 kDa) western blot of LBC1936 and AD samples. c Representative total tau (Tau13; 45-60 kDa) blot, with LBC1936 and AD samples. d Phosphorylated-tau (PHF1; 50-65 kDa) western blot of LBC1936 and AD samples. GAPDH (36 kDa) was run as a loading control for all experiments. Histogram represents the mean of three experimental repeats and the bars represent the quantification of the bands directly above

observed, were similar between both cases (Fig. 13a, b). Furthermore, no differences were observed in any of the parameters analyzed in the post-synapse (Fig. 13a). Intriguingly, the apposition length (the length of the pre- and post-synaptic membrane directly opposing each other) was slightly longer in the AD brain (LBC1936 $=360 \pm 144.5$, $\mathrm{AD}=402.4 \pm 149.6 ; \quad$ Mann-Whitney $U$ test $p=0.006$; Fig. 13a), despite smaller volumes of pre and post synapses detected by array tomography. This increase in apposition length could be a mechanism to try and compensate for shrinking synaptic volume. Electron microscopy can also be used to analyze white matter integrity (Fig. 13c) by measuring axon diameter, myelin thickness and the ratio of both known as the "G-ratio" (Fig. 13d). Measuring the G-ratio from four distinct white matter tracts of the LBC1936 brain revealed good axonal and myelin integrity, with G-ratio values falling remarkably close to the theoretically optimal value of 0.6 [55].
In summary, our electron microscopy approach reveals remarkable synaptic preservation, allowing us to investigate ultrastructural changes in human synapses, post-mortem. We detected changes in pre-synaptic mitochondria and apposition length in our $\mathrm{AD}$ brain and also the presence of degenerating profiles in the LBC1936 brain.

\section{Discussion}

This study has, for the first time, described an experiment spanning almost 70 years, detailing an individual's childhood and ageing cognitive performance and correlating this with in vivo imaging and post-mortem analysis to the level of individual axons and synapses. This proof-of-principle study reinforces the impact of the well-characterized LBC1936 cohort and showcases the depth of anatomical data we can gather post-mortem, to feed into this ever-expanding project, aiming to unravel the physiological processes involved in brain 


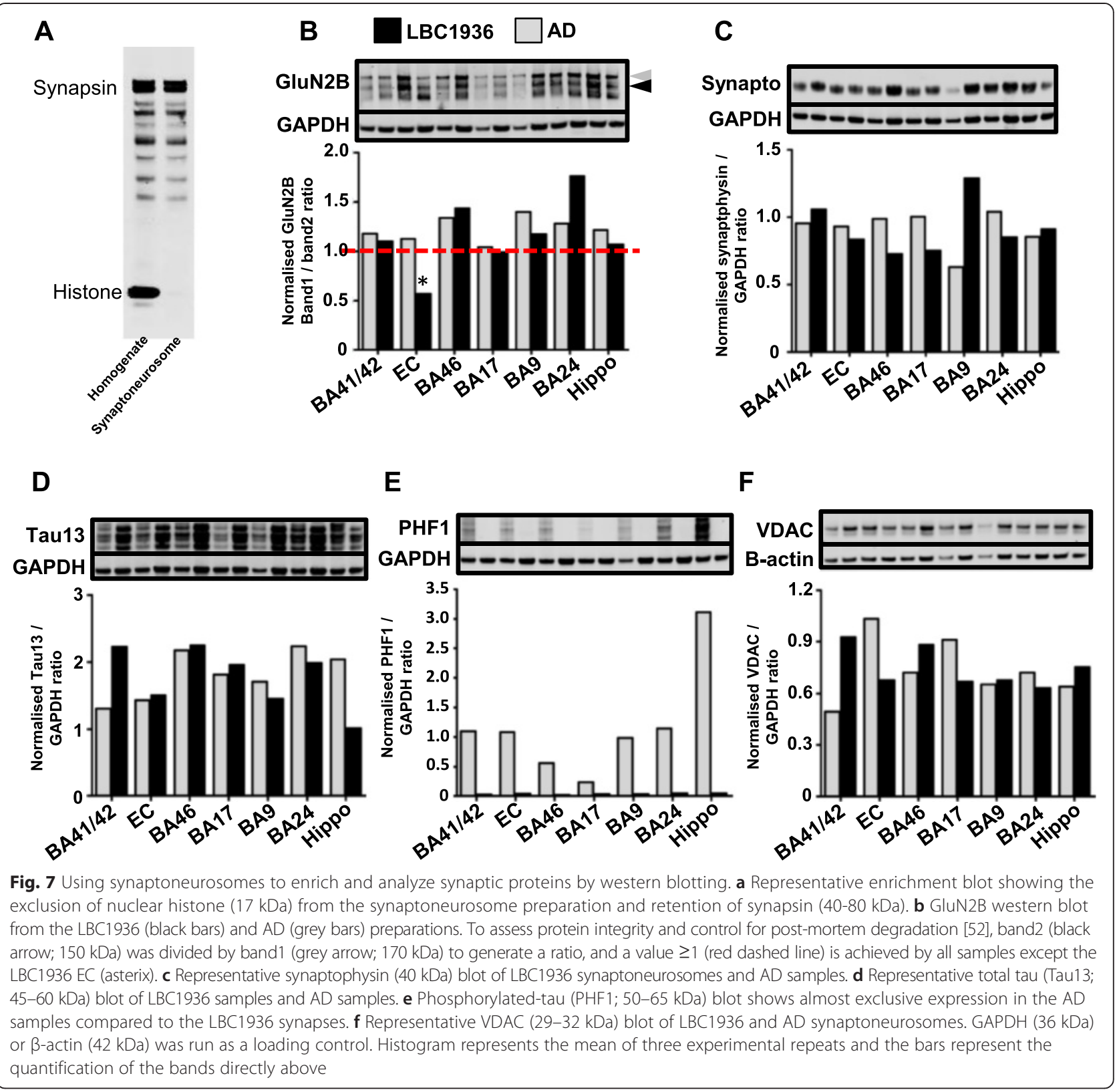

ageing. A significant strength of our protocol is the ability to sample and analyze many distinct cortical, subcortical and white matter regions of the brain, in relative speed, with our biochemical and array tomography approaches. For example, the Religious Orders Study and the Rush Memory and Ageing Project, generally analyze post-mortem pathology in a small number of brain regions (midfrontal gyrus, the superior temporal gyrus, the inferior parietal gyrus, and the entorhinal cortex) and extrapolate global burdens from their average $[15,56]$, thus missing detailed regional variation in brain change. Given the heterogeneous clinical representation of normal and pathological ageing [57, 58], it's critical to assess the underlying brain structure from as wide an array of regions as possible to be able to fully understand the presenting phenotype.

Post-mortem pathological assessment is the only definitive way to confirm a premorbid diagnosis of Alzheimer's disease, however this approach has proved ineffective for categorizing patients with no or mild premorbid cognitive impairment, as pathological burden varies dramatically within these cohorts [59]. This has led to a cognitive reserve theory, in which some people retain impressive cognitive function into old age, despite a heavy pathological load. The hypothesis states that people with high cognitive function throughout life (high IQ, high level of education, extensive literacy and complex social groups) can flexibly and efficiently draw processing power 

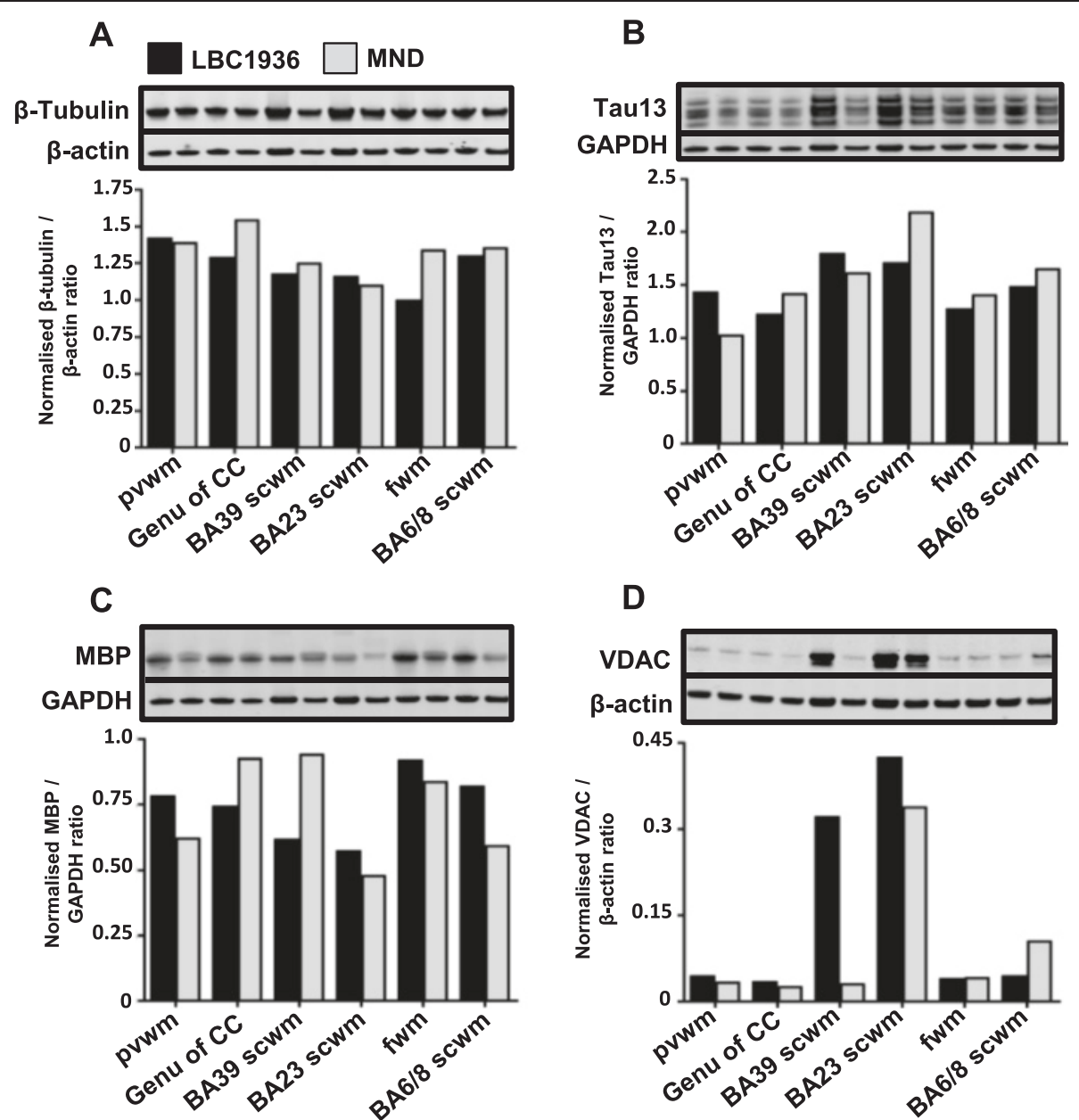

Fig. 8 Assessment of axonal integrity and pathology using crude homogenate western blotting. a Representative $\beta$-III-tubulin (55 kDa) blot of five LBC1936 (black bars) and five MND (grey bars) white matter crude homogenates. b Total tau (Tau13; 45-60 kDa) blot of LBC1936 and MND white matter homogenates. c Representative MBP (23 kDa) blot of LBC1936 and MND homogenates. d VDAC (29-32 kDa) blot of LBC1936 and MND white matter samples. GAPDH $(36 \mathrm{kDa}$ ) or $\beta$-actin $(42 \mathrm{kDa}$ ) was run as a loading control. Histogram represents the mean of three experimental repeats and the bars represent the quantification of the bands directly above

from other brain regions and thus retain general cognitive ability, despite underlying pathology [60]. Some of these traits have been shown to positively influence cognitive ageing in our Lothian Birth Cohort 1936 [17]. One potential strategy to help those people with pathology and cognitive decline is to remove the pathological burden allowing the brain to function more efficiently. This has been the primary goal for Alzheimer's pharmacotherapy for many years, but unfortunately has proved spectacularly unsuccessful with a $99.6 \%$ failure rate [61]. Therefore, new therapeutic strategies are needed and synapses or synaptic function may represent a novel approach as synapse loss is the strongest correlate with cognitive decline [62].

This enforces the importance of studying synapse density, volume and pathology in ageing cohorts such as the LBC1936, as it will undoubtedly provide valuable insight into the processes involved in synapse loss and it's effect on brain structure and function. Our array tomography approach can reliably detect synaptic density differences between our nondemented LBC1936 brain and the AD brain (Fig. 9) and will be an important addition to the LBC1936 study as more tissue becomes available. The addition of more cases will allow us to test the correlation between cognitive performance and synaptic density in the brain regions thought to be important for specific cognitive tasks. Furthermore, synaptic density measurements can be compared with intrinsic pathology load to assess whether an inverse correlation exists or whether synapse loss is independent of detectible pathology. Without having robust data from young brains, we cannot predict how well synaptic density is preserved in the nondemented, aged LBC1936 brain, but we can conclude that 


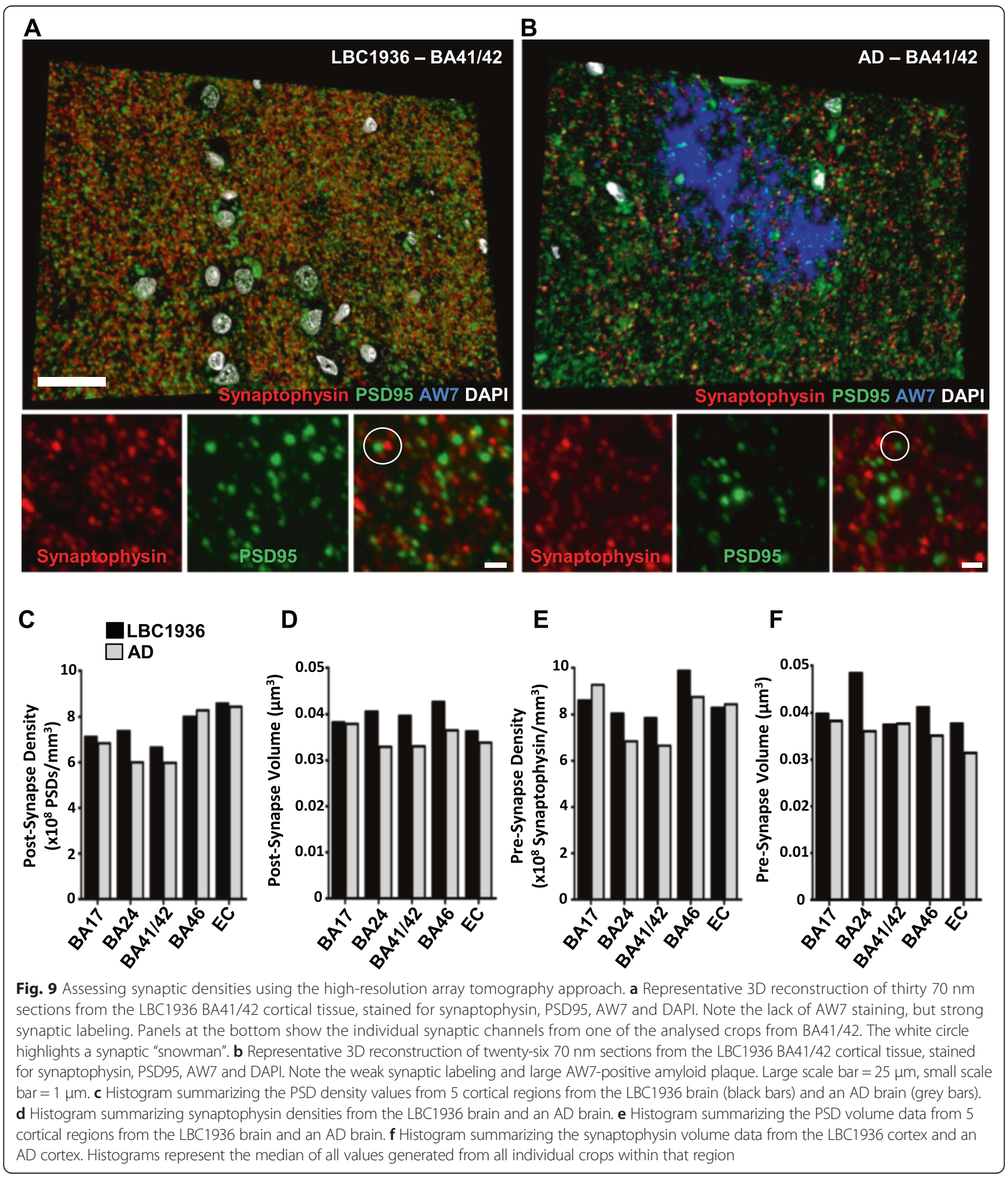

compared to a pathologically aged Alzheimer's brain there appears to be synaptic preservation.

Given the range of visible pathologies in the MRI scans from the LBC1936 cohort (Fig. 2), it will be interesting to see if these affect neuronal connectivity. Does dramatic cortical thinning mean dramatic synapse loss, or does the brain compensate by increasing connections between cells? In a seminal paper on the subject [63] DeKosky and Scheff describe cortical thinning and significant synapse loss in autopsied AD brains, suggesting that these brains don't compensate by increasing synapse number, but interestingly the authors reported an increase in synaptic 

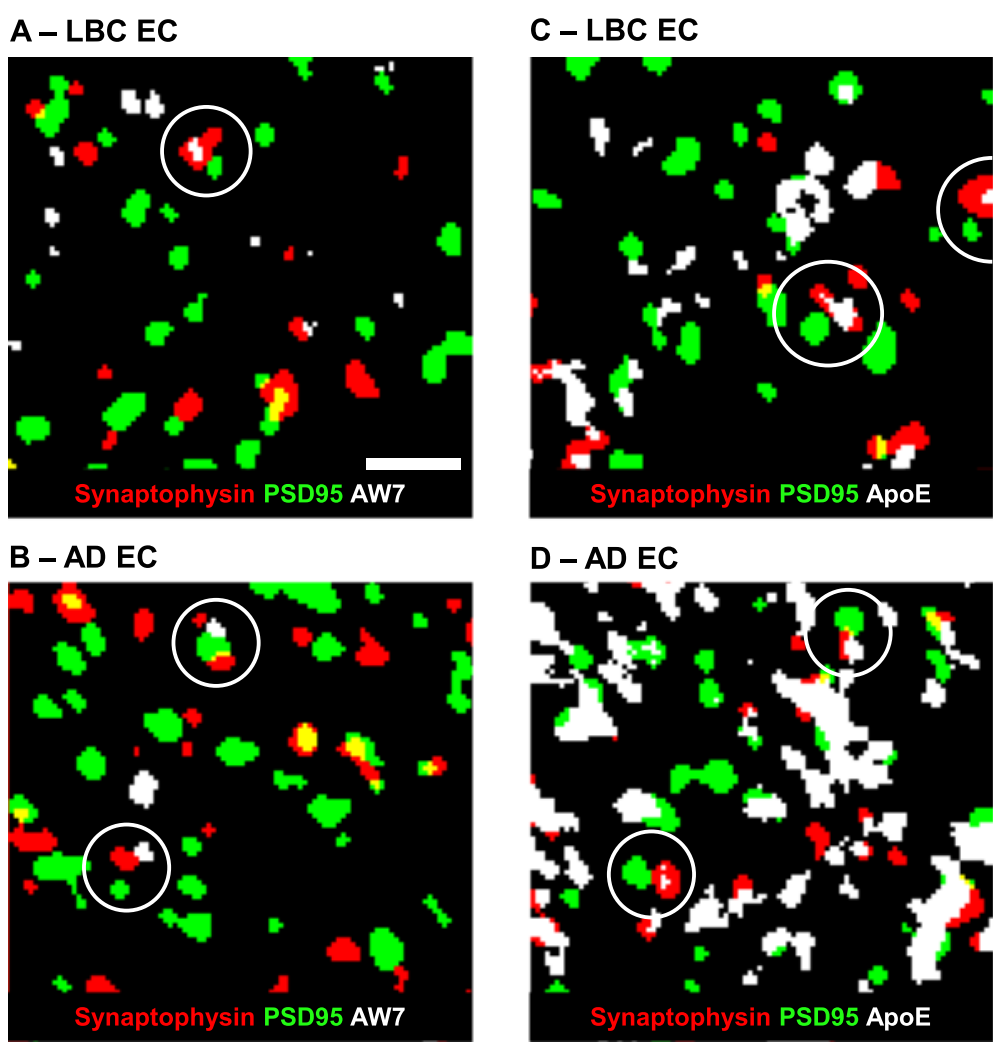

Fig. 10 Using array tomography to assess the presence of synaptotoxic proteins. Representative images from a single region of interest (crop) captured within the LBC1936 EC $(\mathbf{a}+\mathbf{c})$ or the AD EC $(\mathbf{b}+\mathbf{d})$. Each image is a single plane from a 3D stack, which has been thresholded/binarised and single-slice objects removed to eliminate background. Sections were stained for synaptophysin, PSD95, and AW7 (a+b) or ApoE (c + d). Synaptically located staining is highlighted with white circles. c Scale bar $=2 \mu \mathrm{m}$

apposition length. This was also observed in our EM analysis (Fig. 13) but was not replicated in the array experiments revealing a smaller synaptic volume in the $\mathrm{AD}$ samples (Fig. 9). Array tomography cannot resolve ultrastructural detail, such as apposition length, but does allow high-throughput analysis of thousands of synapses and yields information about the protein composition of synapses. The presence of toxic amyloid species in synapses results in shrinkage and synaptic breakdown [64] and given the heavy amyloid burden in the AD brain (Fig. 4) it's likely that some synaptic damage is present, which may explain our array tomography results. Indeed, we find a higher burden of synaptic amyloid in all $\mathrm{AD}$ samples compared to the LBC1936 brain (Fig. 11).

We have previously shown that possessing an e4 allele of the apolipoprotein E (APOE) gene correlates with increased cognitive decline in older age [24] and it confers a higher risk of developing Alzheimer's disease [65]. APOE genotyping revealed the LBC1936 participant was APOE e3/e3 and the $\mathrm{AD}$ case was APOE e3/e4. We have previously shown that the ApoE protein is found in synapses in human brain [54]. Here, we found the LBC1936 cortex contained approximately $25 \%$ of ApoE-positive synapses, compared to approximately $40 \%$ in the AD brain (Fig. 11), strikingly similar to the $35 \%$ of ApoE-positive synapses reported previously [54]. Finally, greater ApoE staining in the $\mathrm{AD}$ case associated with more amyloid at the synapse (Fig. 11). Amyloid was found at a subset of synapses in both cases, however the exact form of amyloid (monomer, polymer or fibril etc.) was not identified. The antibody we used in this study (AW7) labels all forms of amyloid. The most synaptotoxic form has yet to be conclusively revealed, although we have data suggesting multiple oligomeric forms of amyloid are present at synapses (data not shown). All of these findings match our previous results in human brain and demonstrate that our array tomography approach has the ability to reveal synaptic density and protein constitution of human synapses.

Our results show that the cognitively-healthy LBC1936 brain has more synapses than the AD brain and a lower pathological load; however some synapses in the LBC1936 brain contained amyloid, which might predispose them to degeneration. As synapses degenerate the spatial control of proteins is lost and the synapse fills with excess protein 


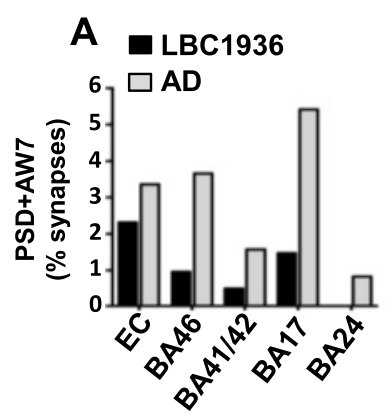

D

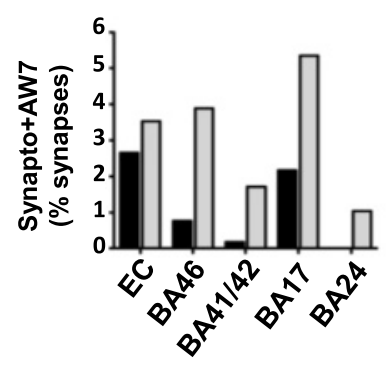

G

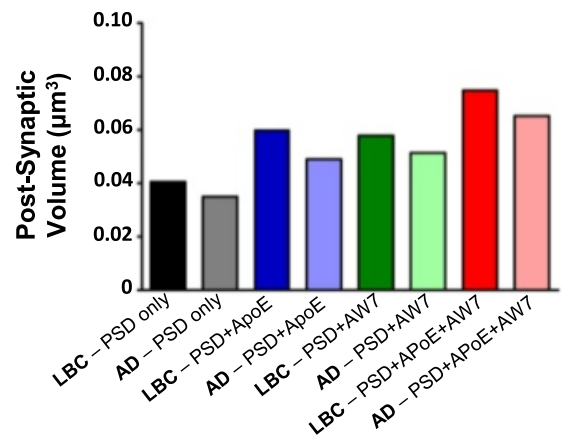

B

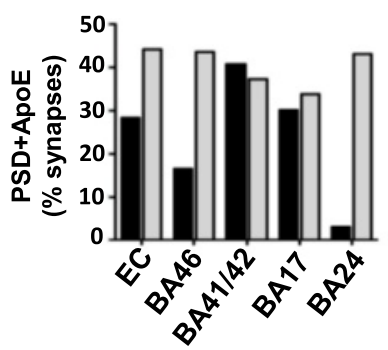

E

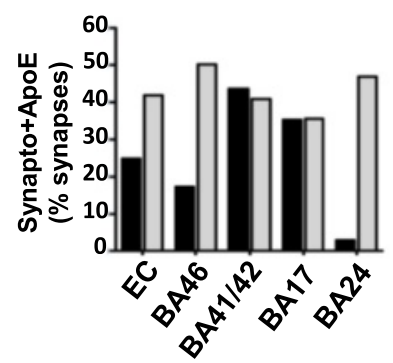

C

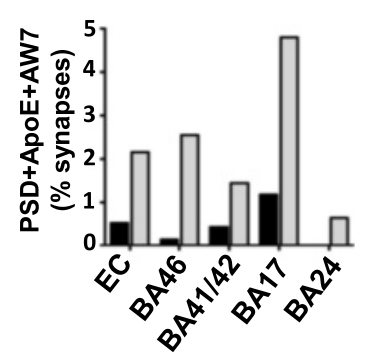

F

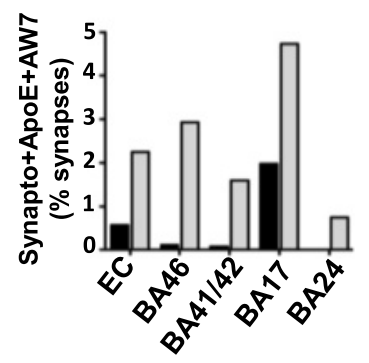

H

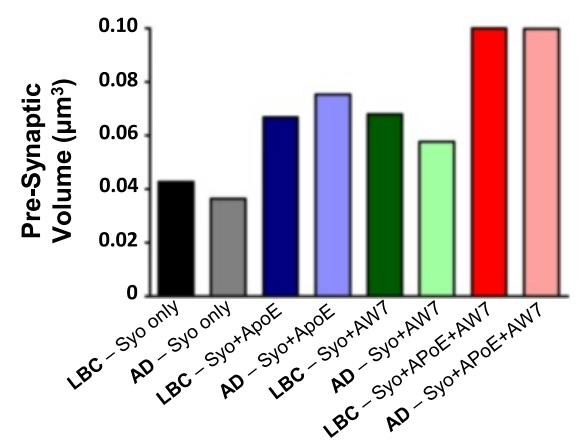

Fig. 11 Analysing the synaptic localization of $\beta$-amyloid and ApoE. a Histogram showing the percentage of synapses that exhibits co-localization between PSD95 and AW7 in the LBC1936 (black bars) and AD (grey bars) samples. The percentage of co-localization between PSD95 and ApoE in the LBC1936 and AD samples is shown in (b) c The percentage of co-localization between PSD95, ApoE and AW7 in the LBC1936 and AD samples. $\mathbf{d}$ Histogram showing the percentage of synapses that exhibits co-localization between synaptophysin and AW7 in the LBC1936 and AD samples. The percentage of co-localization between synaptophysin and ApoE in the LBC1936 and AD samples is shown in (e). f The percentage of co-localization between synaptophysin, ApoE and AW7 in the LBC1936 and AD samples. $g$ Histogram representing the median post-synapse (PSD) volume of all synapses from all cortical regions, positive for PSD alone, or combinations of PSD \pm pathology as noted on $\mathbf{x}$-axis. $\mathbf{h}$ Histogram representing the median pre-synapse (synaptophysin) volume of all synapses from all cortical regions, positive for synaptophysin alone, or combinations of synaptophysin \pm pathology as noted on $x$-axis

and debris, which renders the terminal electron dense under an electron microscope. We discovered a higher incidence of electron dense synapses in the AD brain than the LBC1936 samples, however some degenerating synapses were found in the cognitively-healthy LBC1936 brain. This could represent the small percentage of synapses that contain amyloid from our array tomography studies, which have reached the point of degeneration. However, without a young brain as a control it is difficult to confirm this.
The LBC1936 study has revealed that white matter integrity correlates with cognitive performance [66, 67], therefore detailed analysis of white matter at post-mortem will aid in the identification of visible abnormalities and hyperintensities observed during in vivo imaging (Fig. 2), which are currently unexplained $[68,69]$. Our biochemistry approach allows a quick evaluation of gross protein changes in white matter tracts, which can then be resolved to sub-micron resolution using array tomography, in the hope to correlate in vivo observations with single cell 


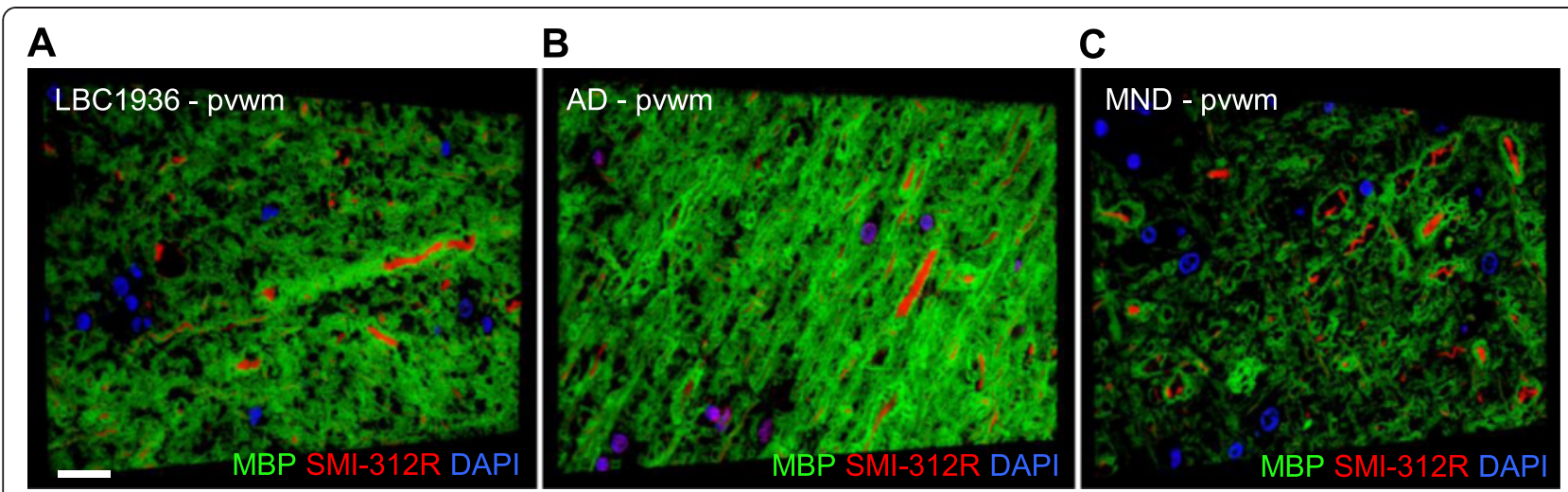

Fig. 12 Assessing white matter integrity with the super-resolution array tomography approach. Representative 3D reconstructions of twenty-five $70 \mathrm{~nm}$ sections from the LBC1936 pvwm tissue (a), twenty-nine $70 \mathrm{~nm}$ sections from the AD pvwm tissue (b) and fourteen 70 nm sections from the MND prwm tissue (c), all stained for SMI-312R, MBP and DAPI. Scale bars $=15 \mu \mathrm{m}$

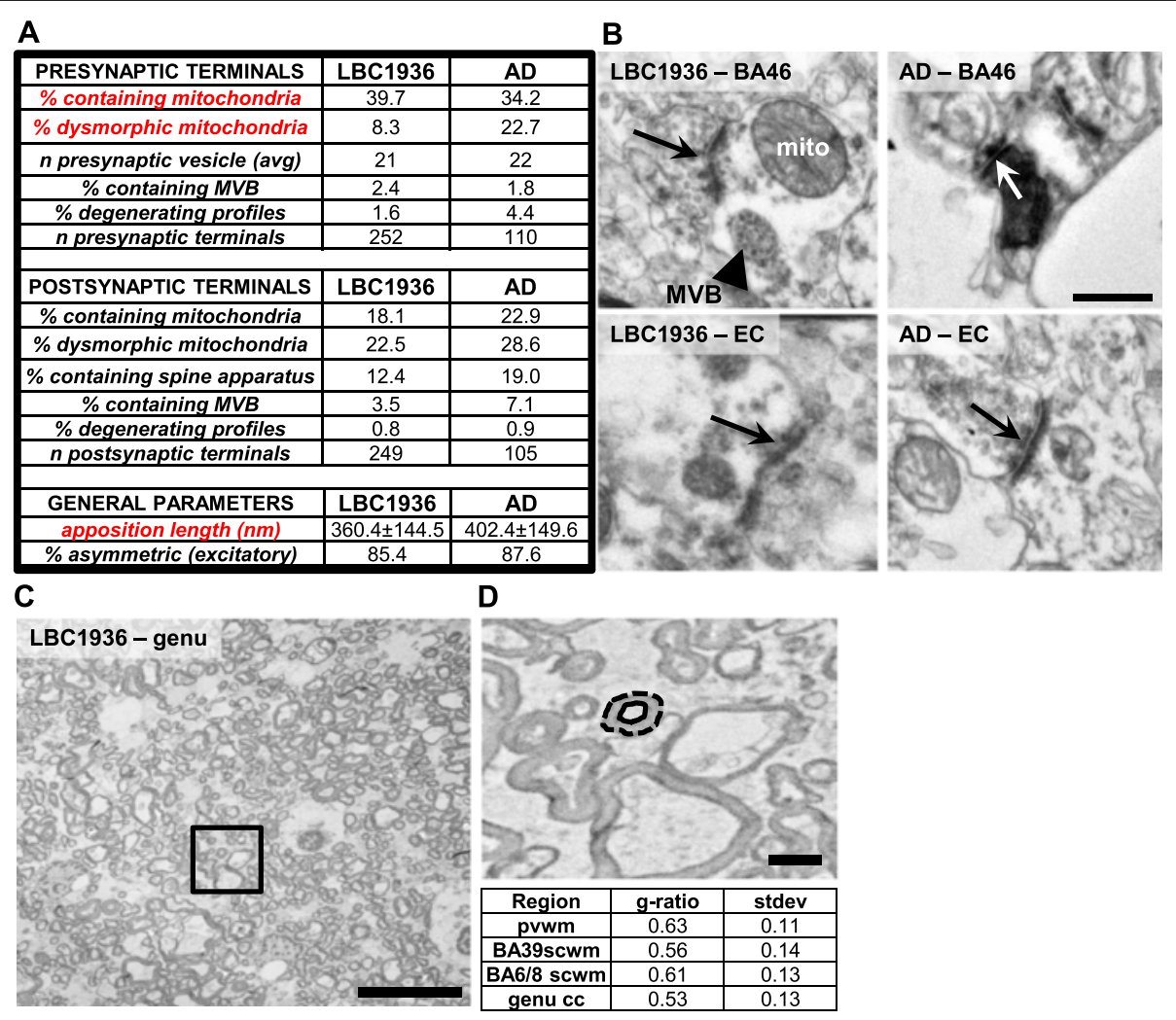

Fig. 13 Assessment of synaptic and axonal ultrastructure using transmission electron microscopy. a. Table summarizing the pre- and post-synaptic parameters measured in the LBC1936 and AD brains at the EM level. Significant differences are highlighted in red. b. Representative electron micrographs from BA46 and EC from the LBC1936 and AD brains. Arrows point to the synapse from the pre-synapse. White arrow highlights an electron dense degenerating synapse. Mito = mitochondria, MVB = multi-vesicular body. c. Representative electron micrograph from the LBC1936 genu of the corpus callosum sample, showing axons cut in cross-section. $\mathbf{d}$. Higher magnification of the boxed section in (c), showing how $\mathrm{g}$-ratio is determined in each sample. The perimeter is draw around the internal axon cross-section (solid line) and divided by the perimeter of the axon + myelin cross-section (dashed line). G-ratio values from four white matter regions are found in the adjoining table 
alterations in protein composition. As shown in Fig. 8, MBP levels were quite variable between regions and cases. This could represent interesting differences in clinical and sub-clinical alterations in myelin physiology, or could be explained by post-mortem degradation of MBP [70]. Further work is required to more accurately interpret these findings and ensure we use the correct measures of axonal integrity in future studies. Array tomography can build a useful 3D representation of axons and surrounding myelin to assess the spatial distribution of key proteins in axonal integrity. For example, in future studies we can accurately measure numerous paranodal proteins found in the critical region adjacent to the Nodes of Ranvier, containing clustered ion channels and adhesion proteins, which is a vulnerable region following axonal injury or stress [71]. Electron microscopy reveals the structure of membranes in exquisite detail and can be used to calculate axonal myelin coverage. It has been predicted that the ideal G-ratio should be 0.6 [55] and we found in our LBC1936 samples that G-ratios from distinct white matter regions were remarkably close to this figure. This suggests our LBC1936 has good myelin integrity and seems to associate well with their strong cognitive performance. Greater understanding of white matter integrity in ageing brains will significantly enhance our understanding of age-related brain dysfunction and may lead to alternative therapeutics for prolonging cognition into older age.

The Edinburgh Brain Bank is currently in the process of generating a grading system for characterizing the extent of small vessel disease and different types of infarcts (microinfarcts, large vessel infarcts and lacunar infarcts). Cognitive decline correlates strongly with the presence of underlying cerebrovascular disease [72, 73] and given the history of stroke in our LBC1936 patient in their later years and the presence of small old infarcts in the MR images, future characterization of all cases will involve assessment of the vasculature integrity and presence of localized infarcts. This will provide valuable information on the role of the cerebral vasculature on brain function and significantly strengthen the academic potential of the LBC1936 study.

\section{Conclusion}

Ultimately, this study showcases the remarkable wealth of information we can generate post-mortem from the LBC1936 donors. As more tissue becomes available we will be able to draw unique correlations between underlying pathology to the level of individual synapses with premorbid longitudinal cognitive performance. Along with the extensive biochemical, histopathological and imaging data here, genetic factors, biomarkers and psycho-social factors can all be obtained from individuals to drive real progress in our understanding of the factors involved in normal cognitive ageing.

\section{Additional files}

Additional file 1: Figure S1. Summary of the array tomography protocol. Numbered step-by-step flowchart summarizing the steps involved in performing array tomography. Loosely separated into three sections; tissue preparation (blue), imaging (green) and image analysis (orange). A full, detailed protocol can be found in [34]. All ImageJ and MATLAB scripts are available on request. (PPTX $19 \mathrm{~kb}$ )

Additional file 2: Figure S2. Reference key for the semi-quantitative neuropathology scoring. Representative images showing the range of staining observed in the brains of our two cases. $\mathrm{H}+\mathrm{E}$ stain is shown to highlight the clear border between the grey and white matter (white arrows), which was used to calculate cortical thickness. BA4 "score -" represents no staining. Sections were given a "score + " if any plaques were found. Scores " ++ " and " +++ " represent clear increases in amyloid burden. AT8 "score -" represents no staining. Sections were given a "score +" if AT8-positive neurites were found, but no somatic tangles. Score "+ $+"$ represents strong neuritic staining and a small number of somatic tangles. Score " +++ " represents strong diffuse neuritic labeling and frequent somatic tangles. TDP-43 "score -" is given if the vast majority of cells express TDP-43 only in the nucleus. Score " + " is given if the majority of cells express TDP-43 in the nucleus, but also diffuse cytoplasmic labeling. Score " ++ " means the nucleus is mostly clear of TDP-43 staining and strong, diffuse cytoplasmic staining is evident. Score " +++ " is given if the nucleus is completely clear and the cytoplasm contains strongly labeled aggregates. CD68 is not scored as "-" because microglia are always present. Score " +" represents numerous CD68+ cells scattered through the section. Score " ++ " is given if larger, complex cells with very dark staining are found. Score "++ $+"$ is given if the section contains a majority of large, complex and heavily stained cells. GFAP is not scored as "-" because astrocytes are always present. Score " + " represents diffuse but weak staining of astrocytic processes and cell soma. Score " ++ " is given if a few strongly labeled cell soma are evident, along with heavily labeled processes. Score " +++ " is given when a larger number of strongly labeled cells are present. Ubiquitin is not scored as "-" because ubiquitin is always present. Score " + " represents the appearance of small cellular inclusions. Score "++" is given if ubiquitin-positive tangles are found in some cell soma and neurites. Score " +++ " is given if strongly labeled cellular aggregates are present. Large scale bars $=150 \mu \mathrm{m}$ and inset bars $=30 \mu \mathrm{m}$. (PPTX $16598 \mathrm{~kb})$

\section{Competing interests}

The authors declare that they have no competing interests.

\section{Authors' contributions}

$\mathrm{CMH}$ contributed to experimental design, performed array tomography experiments and analysis, performed neuropathology analysis, prepared all final figures and wrote the manuscript. RJJ performed the western blotting and analysis. JMK contributed to the neuropathology analysis. AGH contributed to the array tomography study design and western blotting experiments and analysis. AKW and TGH designed and performed electron microscopy experiments and analysis. SRC performed and IJD analysed the cognitive profiling, and JMS the medical profiling. IJD recruited participants, heads the in vivo LBC1936 cohort studies, analyzed cognitive data, and contributed to experimental design and writing the manuscript. IJD, SEH and JMS set up the Lothian Birth Cohort 1936 Brain Tissue Bank. MEB and JMW performed and analysed the MRI experiments. CS and CM collected and prepared the tissue for all post-mortem experiments. TLS designed experiments, contributed to array tomography analysis, performed electron microscopy experiments and analysis, and contributed to writing the manuscript. All authors read and approved the final manuscript.

\section{Acknowledgements}

This work was funded by Alzheimer's Research UK/Chief Scientist's Office, Scotland, and a University of Edinburgh (UoE) Wellcome Trust Institutional Strategic Support Fund. Phenotype collection in the Lothian Birth Cohort 1936 was supported by Age UK (The Disconnected Mind project). UoE Centre for Cognitive Ageing and Cognitive Epidemiology (CCACE) is supported by funding from the UK's Biotechnology and Biological Sciences Research Council (BBSRC) and the Medical Research Council (MRC), and the UoE as part of the cross-council Lifelong Health and Wellbeing initiative (MR/K026992/1). The Edinburgh Brain 
Bank is supported by the MRC. The scanning was performed in the Brain Research Imaging Centre (JMW, MEB), which is supported by the Scottish Imaging Network A Platform for Scientific Excellence (SINAPSE) Initiative of the Scottish Funding Council. JMW received funding from the Scottish Funding Council and Chief Scientist Office of the Scottish Government and the Row Fogo Charitable Trust for this work We wish to thank our participants and their families for their generous donations, Peter Davies (Einstein College of Medicine) and Dominic Walsh (Brigham and Women's Hospital and Harvard Medical School) for providing antibodies, Marian DiFiglia and the Philly Dake Electron microscopy core for use of the transmission electron microscope, and the Sylvia Aitken Charitable Trust for support.

\section{Author details}

'Centre for Cognitive and Neural Systems, University of Edinburgh, 1 George Square, Edinburgh EH8 9JZ, UK. ${ }^{2}$ Centre for Integrative Physiology, University of Edinburgh, Hugh Robson Building, George Square, Edinburgh EH8 9XD, UK. ${ }^{3}$ Centre for Cognitive Ageing and Cognitive Epidemiology, University of Edinburgh, 7 George Square, Edinburgh EH8 9JZ, UK. ${ }^{4}$ Medical Genetics Section, University of Edinburgh Centre for Genomic and Experimental Medicine and MRC Institute of Genetics and Molecular Medicine, Western General Hospital, Edinburgh EH4 2XU, UK. ${ }^{5}$ Centre for Clinical Brain Sciences, University of Edinburgh, Chancellor's Building, 49 Little France Crescent, Edinburgh EH16 4SB, UK. ${ }^{6}$ Geriatric Medicine Unit, University of Edinburgh, Western General Hospital, Edinburgh EH4 2XU, UK. ${ }^{7}$ Department of Psychology, CCACE, University of Edinburgh, 7 George Square, Edinburgh EH8 9JZ, UK. ${ }^{8}$ Euan MacDonald Centre for Motor Neurone Disease Research, University of Edinburgh, Chancellor's Building, 49 Little France Crescent, Edinburgh EH16 4SB, UK.

Received: 19 August 2015 Accepted: 19 August 2015

Published online: 04 September 2015

\section{References}

1. Prince M, Matthew P, Maelenn G (2013) World alzheimer report 2013. Journal of caring. An analysis of long-term care for dementia. Health Service and Population Research Department, King's College London, London, http://www.alz.co.uk/sites/default/files/pdfs/WorldAlzheimerReport2013.pdf

2. Cracknell R (2010) The ageing population. Key Issues for the New Parliament 2010. House of Commons Library Research. http://www.parliament.uk/ documents/commons/lib/research/key_issues/Key-lssues-The-ageingpopulation2007.pdf

3. Bishop NA, Lu T, Yankner BA (2010) Neural mechanisms of ageing and cognitive decline. Nature 464(7288):529-535

4. Blazer DG, Yaffe K, Liverman CT (2015) In: C.o.t.P.H.D.o.C. Aging, B.o.H.S Policy, and I.o. Medicine (ed) Cognitive aging: progress in understanding and opportunities for action. National Academy of Sciences, Washington DC

5. Mrak RE, Griffin ST, Graham DI (1997) Aging-associated changes in human brain. J Neuropathol Exp Neurol 56(12):1269-1275

6. Andrews-Hanna JR, Snyder AZ, Vincent JL, Lustig C, Head D, Raichle ME, Buckner RL (2007) Disruption of large-scale brain systems in advanced aging. Neuron 56(5):924-935

7. Valdes Hernandez Mdel C, Booth T, Murray C, Gow AJ, Penke L, Morris Z, Maniega SM, Royle NA, Aribisala BS, Bastin ME, Starr JM, Deary IJ, Wardlaw JM (2013) Brain white matter damage in aging and cognitive ability in youth and older age. Neurobiol Aging 34(12):2740-2747

8. Morrison JH, Baxter MG (2012) The ageing cortical synapse: hallmarks and implications for cognitive decline. Nat Rev Neurosci 13(4):240-250

9. Loerch PM, Lu T, Dakin KA, Vann JM, Isaacs A, Geula C Wang J, Pan Y, Gabuzda DH, Li C, Prolla TA, Yankner BA (2008) Evolution of the aging brain transcriptome and synaptic regulation. PLoS One 3(10): e3329

10. Yankner BA, Lu T, Loerch P (2008) The aging brain. Annu Rev Pathol 3:41-66

11. Honer WG, Barr AM, Sawada K, Thornton AE, Morris MC, Leurgans SE, Schneider JA, Bennett DA (2012) Cognitive reserve, presynaptic proteins and dementia in the elderly. Transl Psychiatry 2: e114

12. Hill WD, Davies G, van de Lagemaat LN, Christoforou A, Marioni RE, Fernandes CP, Liewald DC, Croning MD, Payton A, Craig LC, Whalley L, Horan M, Ollier W, Hansell NK, Wright MJ, Martin NG, Montgomery GW, Steen VM, Le Hellard S, Espeseth T, Lundervold AJ, Reinvang I, Starr JM, Pendleton N, Grant SG, Bates TC, Deary IJ (2014) Human cognitive ability is influenced by genetic variation in components of postsynaptic signalling complexes assembled by NMDA receptors and MAGUK proteins. Transl Psychiatry 4: e341

13. Selkoe DJ (2002) Alzheimer's disease is a synaptic failure. Science 298(5594):789-791

14. Spires-Jones TL, Hyman BT (2014) The intersection of amyloid beta and tau at synapses in Alzheimer's disease. Neuron 82(4):756-771

15. Bennett DA, Wilson RS, Arvanitakis Z, Boyle PA, de Toledo-Morrell L, Schneider JA (2013) Selected findings from the Religious Orders Study and Rush Memory and Aging Project. J Alzheimers Dis 33(Suppl 1):S397-S403

16. Guralnik JM, Kritchevsky SB (2010) Translating research to promote healthy aging: the complementary role of longitudinal studies and clinical trials. J Am Geriatr Soc 58(Suppl 2):S337-S342

17. Deary IJ, Gow AJ, Pattie A, Starr JM (2012) Cohort profile: the Lothian Birth Cohorts of 1921 and 1936. Int J Epidemiol 41(6):1576-1584

18. Bak TH, Nissan JJ, Allerhand MM, Deary IJI (2014) Does bilingualism influence cognitive aging? Ann Neurol 75(6):959-963

19. Ritchie SJ, Bates TC, Deary IJ (2015) Is education associated with improvements in general cognitive ability, or in specific skills? Dev Psychol 51(5):573-582

20. Smart EL, Gow AJ, Deary IJ (2014) Occupational complexity and lifetime cognitive abilities. Neurology 83(24):2285-2291

21. Gow AJ, Corley J, Starr JM, Deary IJ (2013) Which social network or support factors are associated with cognitive abilities in old age? Gerontology 59(5):454-463

22. Gow AJ, Corley J, Starr JM, Deary IJ (2012) Reverse causation in activitycognitive ability associations: the Lothian Birth Cohort 1936. Psychol Aging 27(1):250-255

23. McIntosh AM, Gow A, Luciano M, Davies G, Liewald DC, Harris SE, Corley J, Hall J, Starr JM, Porteous DJ, Tenesa A, Visscher PM, Deary IJ (2013) Polygenic risk for schizophrenia is associated with cognitive change between childhood and old age. Biol Psychiatry 73(10):938-943

24. Luciano M, Gow AJ, Harris SE, Hayward C, Allerhand M, Starr JM, Visscher PM, Deary IJ (2009) Cognitive ability at age 11 and 70 years, information processing speed, and APOE variation: the Lothian Birth Cohort 1936 study. Psychol Aging 24(1):129-138

25. Corley J, Jia X, Brett CE, Gow AJ, Starr JM, Kyle JA, McNeill G, Deary IJ (2011) Alcohol intake and cognitive abilities in old age: the Lothian Birth Cohort 1936 study. Neuropsychology 25(2):166-175

26. Corley J, Gow AJ, Starr JM, Deary IJ (2010) Is body mass index in old age related to cognitive abilities? The Lothian Birth Cohort 1936 Study. Psychol Aging 25(4):867-875

27. Corley J, Starr JM, McNeill G, Deary IJ (2013) Do dietary patterns influence cognitive function in old age? Int Psychogeriatr 25(9):1393-1407

28. Luciano M, Marioni RE, Gow AJ, Starr JM, Deary IJ (2009) Reverse causation in the association between C-reactive protein and fibrinogen levels and cognitive abilities in an aging sample. Psychosom Med 71(4):404-409

29. Gow AJ, Firth CM, Harrison R, Starr JM, Moss P, Deary IJ (2013) Cytomegalovirus infection and cognitive abilities in old age. Neurobiol Aging 34(7):1846-1852

30. Penke L, Maniega SM, Bastin ME, Valdes Hernandez MC, Murray C, Royle NA, Starr JM, Wardlaw JM, Deary IJ (2012) Brain white matter tract integrity as a neural foundation for general intelligence. Mol Psychiatry 17(10):1026-1030

31. Royle NA, Booth T, Valdes Hernandez MC, Penke L, Murray C, Gow AJ, Maniega SM, Starr J, Bastin ME, Deary IJ, Wardlaw JM (2013) Estimated maximal and current brain volume predict cognitive ability in old age. Neurobiol Aging 34(12):2726-2733

32. Penke L, Valdes Hernandez MC, Maniega SM, Gow AJ, Murray C, Starr JM, Bastin ME, Deary IJ, Wardlaw JM (2012) Brain iron deposits are associated with general cognitive ability and cognitive aging. Neurobiol Aging 33(3):510-517, e2

33. Karama S, Bastin ME, Murray C, Royle NA, Penke L, Munoz Maniega S, Gow AJ, Corley J, Valdes Hernandez Mdel C, Lewis JD, Rousseau ME, Lepage C, Fonov V, Collins DL, Booth T, Rioux P, Sherif T, Adalat R, Starr JM, Evans AC, Wardlaw JM, Deary IJ (2014) Childhood cognitive ability accounts for associations between cognitive ability and brain cortical thickness in old age. Mol Psychiatry 19(5):555-559

34. Kay KR, Smith C, Wright AK, Serrano-Pozo A, Pooler AM, Koffie R, Bastin ME, Bak TH, Abrahams S, Kopeikina KJ, McGuone D, Frosch MP, Gillingwater TH, Hyman BT, Spires-Jones TL (2013) Studying synapses in human brain with array tomography and electron microscopy. Nat Protoc 8(7):1366-1380

35. Deary IJ, Gow AJ, Taylor MD, Corley J, Brett C, Wilson V, Campbell H, Whalley LJ, Visscher PM, Porteous DJ, Starr JM (2007) The Lothian Birth 
Cohort 1936: a study to examine influences on cognitive ageing from age 11 to age 70 and beyond. BMC Geriatr 7:28

36. Wardlaw JM, Bastin ME, Valdes Hernandez MC, Maniega SM, Royle NA, Morris Z, Clayden JD, Sandeman EM, Eadie E, Murray C, Starr JM, Deary IJ (2011) Brain aging, cognition in youth and old age and vascular disease in the Lothian Birth Cohort 1936: rationale, design and methodology of the imaging protocol. Int J Stroke 6(6):547-559

37. Samarasekera N, Al-Shahi Salman R, Huitinga I, Klioueva N, McLean CA Kretzschmar H, Smith C, Ironside JW (2013) Brain banking for neurological disorders. Lancet Neurol 12(11):1096-1105

38. Tai HC, Serrano-Pozo A, Hashimoto T, Frosch MP, Spires-Jones TL, Hyman BT (2012) The synaptic accumulation of hyperphosphorylated tau oligomers in Alzheimer disease is associated with dysfunction of the ubiquitinproteasome system. Am J Pathol 181(4):1426-1435

39. Geinisman Y, Gundersen HJ, van der Zee E, West MJ (1996) Unbiased stereological estimation of the total number of synapses in a brain region. J Neurocytol 25(12):805-819

40. Roche SL, Sherman DL, Dissanayake K, Soucy G, Desmazieres A, Lamont DJ, Peles E, Julien JP, Wishart TM, Ribchester RR, Brophy PJ, Gillingwater TH (2014) Loss of glial neurofascin155 delays developmental synapse elimination at the neuromuscular junction. J Neurosci 34(38):12904-12918

41. Salat DH, Buckner RL, Snyder AZ, Greve DN, Desikan RS, Busa E, Morris JC, Dale AM, Fischl B (2004) Thinning of the cerebral cortex in aging. Cereb Cortex 14(7):721-730

42. Debette S, Markus HS (2010) The clinical importance of white matter hyperintensities on brain magnetic resonance imaging: systematic review and meta-analysis. BMJ 341:c3666

43. Price JL, Morris JC (1999) Tangles and plaques in nondemented aging and "preclinical" Alzheimer's disease. Ann Neurol 45(3):358-368

44. Price JL, McKeel DW, Jr., Buckles VD, Roe CM, Xiong C, Grundman M, Hansen LA, Petersen RC, Parisi JE, Dickson DW, Smith CD, Davis DG, Schmitt FA, Markesbery WR, Kaye J, Kurlan R, Hulette C, Kurland BF, Higdon R, Kukull W, Morris JC (2009) Neuropathology of nondemented aging: presumptive evidence for preclinical Alzheimer disease. Neurobiol Aging 30(7):1026-1036

45. Vinters HV, Gilbert JJ (1983) Cerebral amyloid angiopathy: incidence and complications in the aging brain. II. The distribution of amyloid vascular changes. Stroke 14(6):924-928

46. Pekny M, Nilsson M (2005) Astrocyte activation and reactive gliosis. Glia 50(4):427-434

47. Mackenzie IR, Rademakers R, Neumann M (2010) TDP-43 and FUS in amyotrophic lateral sclerosis and frontotemporal dementia. Lancet Neurol 9(10):995-1007

48. Deger JM, Gerson JE, Kayed R (2015) The interrelationship of proteasome impairment and oligomeric intermediates in neurodegeneration. Aging Cell. doi:10.1111/acel

49. Kim WS, Kagedal K, Halliday GM (2014) Alpha-synuclein biology in Lewy body diseases. Alzheimers Res Ther 6(5):73

50. Middeldorp J, Hol EM (2011) GFAP in health and disease. Prog Neurobiol 93(3):421-443

51. Whitehouse PJ, Price DL, Struble RG, Clark AW, Coyle JT, Delon MR (1982) Alzheimer's disease and senile dementia: loss of neurons in the basal forebrain. Science 215(4537):1237-1239

52. Bayés À, Collins MO, Galtrey CM, Simonnet C, Roy M, Croning MD et al (2014) Human post-mortem synapse proteome integrity screening for proteomic studies of postsynaptic complexes. Mol Brain 7:88

53. Buee L, Bussiere T, Buee-Scherrer V, Delacourte A, Hof PR (2000) Tau protein isoforms, phosphorylation and role in neurodegenerative disorders. Brain Res Brain Res Rev 33(1):95-130

54. Koffie RM, Hashimoto T, Tai HC, Kay KR, Serrano-Pozo A, Joyner D, Hou S, Kopeikina KJ, Frosch MP,Lee VM, Holtzman DM, Hyman BT, Spires-Jones TL (2012) Apolipoprotein E4 effects in Alzheimer's disease are mediated by synaptotoxic oligomeric amyloid- $\beta$. Brain 135(Pt 7):2155-2168

55. Rushton WA (1951) A theory of the effects of fibre size in medullated nerve. J Physiol 115(1):101-122

56. Bennett DA, Wilson RS, Schneider JA, Evans DA, Aggarwal NT, Arnold SE, Cochran EJ, Berry-Kravis E, Bienias UL (2003) Apolipoprotein E epsilon4 allele, AD pathology, and the clinical expression of Alzheimer's disease. Neurology 60(2):246-252

57. Dubois B, Feldman HH, Jacova C, Cummings $J$, Dekosky ST, BarbergerGateau P, Delacourte A, Frisoni G, Fox NC, Galasko D, Gauthier S, Hampel H, Jicha GA, Meguro K, O'Brien J, Pasquier F, Robert P, Rossor M, Salloway S,
Sarazin M, de Souza LC, Stern Y, Visser PJ, Scheltens P (2010) Revising the definition of Alzheimer's disease: a new lexicon. Lancet Neurol 9(11):11181127

58. Levy R (1994) Aging-associated cognitive decline. Working Party of the International Psychogeriatric Association in collaboration with the World Health Organization. Int Psychogeriatr 6(1):63-68

59. Tucker AM, Stern Y (2011) Cognitive reserve in aging. Curr Alzheimer Res 8(4):354-60

60. Stern $Y$ (2012) Cognitive reserve in ageing and Alzheimer's disease. Lancet Neurol 11(11):1006-1012

61. Cummings $\mathrm{J}$, Morstorf T, Zhong K (2014) Alzheimer's disease drugdevelopment pipeline: few candidates, frequent failures. Alzheimers Res Ther 6(4):37

62. Arendt T (2009) Synaptic degeneration in Alzheimer's disease. Acta Neuropathol 118(1):167-179

63. DeKosky ST, Scheff SW (1990) Synapse loss in frontal cortex biopsies in Alzheimer's disease: correlation with cognitive severity. Ann Neurol 27(5):457-464

64. Koffie RM, Hyman BT, Spires-Jones TL (2011) Alzheimer's disease: synapses gone cold. Mol Neurodegener 6(1):63

65. Corder EH, Saunders AM, Strittmatter WJ, Schmechel DE, Gaskell PC, Small GW, Roses AD, Haines JL, Pericak-Vance MA (1993) Gene dose of apolipoprotein E type 4 allele and the risk of Alzheimer's disease in late onset families. Science 261(5123):921-923

66. Penke L, Munoz Maniega S, Murray C, Gow AJ, Hernandez MC, Clayden JD, Starr JM, Wardlaw JM, Bastin ME, Deary IJ (2010) A general factor of brain white matter integrity predicts information processing speed in healthy older people. J Neurosci 30(22):7569-7574

67. Ritchie SJ, Bastin ME, Tucker-Drob EM, Maniega SM, Engelhardt LE, Cox SR, Royle NA, Gow AJ, Corley J, Pattie A, Taylor AM, Valdes Hernandez Mdel C, Starr JM, Wardlaw JM, Deary IJ (2015) Coupled changes in brain white matter microstructure and fluid intelligence in later life. J Neurosci 35(22):8672-8682

68. Brickman AM (2013) Contemplating Alzheimer's disease and the contribution of white matter hyperintensities. Curr Neurol Neurosci Rep 13(12):415

69. Brickman AM, Muraskin J, Zimmerman ME (2009) Structural neuroimaging in Altheimer's disease: do white matter hyperintensities matter? Dialogues Clin Neurosci 11(2):181-190

70. Barker R, Wellington D, Esiri MM, Love S (2013) Assessing white matter ischemic damage in dementia patients by measurement of myelin proteins. J Cereb Blood Flow Metab 33(7):1050-1057

71. Coleman M (2005) Axon degeneration mechanisms: commonality amid diversity. Nat Rev Neurosci 6(11):889-898

72. Pantoni $L$ (2010) Cerebral small vessel disease: from pathogenesis and clinical characteristics to therapeutic challenges. Lancet Neurol 9(7):689-701

73. O'Brien J, Erkinjuntti T, Reisberg B, Roman G, Sawada T, Pantoni L, Bowler JV Ballard C, DeCarli C, Gorelick PB, Rockwood K, Burns A, Gauthier S, DeKosky ST (2003) Vascular cognitive impairment. Lancet Neurol 2(2):89-98.

\section{Submit your next manuscript to BioMed Central and take full advantage of:}

- Convenient online submission

- Thorough peer review

- No space constraints or color figure charges

- Immediate publication on acceptance

- Inclusion in PubMed, CAS, Scopus and Google Scholar

- Research which is freely available for redistribution 